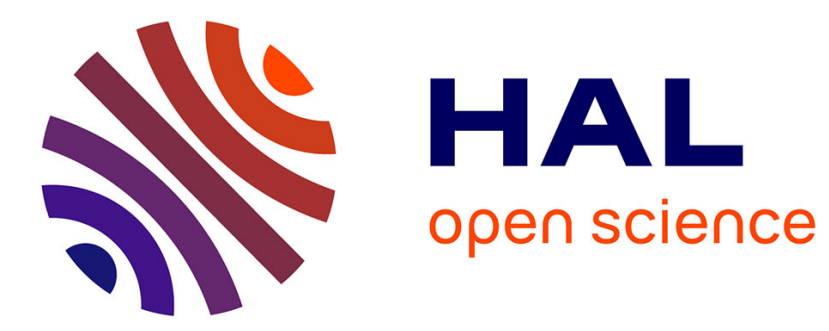

\title{
Kinematic and dynamic analysis of lower-mobility cooperative arms
}

\author{
Philip Long, Wisama Khalil, Stéphane Caro
}

\section{To cite this version:}

Philip Long, Wisama Khalil, Stéphane Caro. Kinematic and dynamic analysis of lower-mobility cooperative arms. Robotica, 2014, pp.1 - 22. 10.1017/S0263574714001039 . hal-01084563

\section{HAL Id: hal-01084563 https://hal.science/hal-01084563}

Submitted on 19 Nov 2014

HAL is a multi-disciplinary open access archive for the deposit and dissemination of scientific research documents, whether they are published or not. The documents may come from teaching and research institutions in France or abroad, or from public or private research centers.
L'archive ouverte pluridisciplinaire HAL, est destinée au dépôt et à la diffusion de documents scientifiques de niveau recherche, publiés ou non, émanant des établissements d'enseignement et de recherche français ou étrangers, des laboratoires publics ou privés. 


\title{
Kinematic and Dynamic Analysis of Lower-Mobility Cooperative Arms
}

\author{
Philip Long $^{\nabla \diamond} \quad$ Wisama Khalil $^{{ }^{\diamond}} \quad$ Stéphane Caro $^{\diamond *}$ \\ $\nabla$ LUNAM, Ecole Centrale de Nantes, 1 rue de la Noë, 44321 Nantes, France \\ $\diamond \mathrm{IRCCyN}_{\text {, UMR CNRS n }}^{\circ}$ 6597, 1 rue de la Noë, 44321 Nantes, France \\ Email addresses: philip.long@irccyn.ec-nantes.fr, \\ wisama.khalil@irccyn.ec-nantes.fr, stephane.caro@irccyn.ec-nantes.fr
}

\begin{abstract}
This paper studies the modeling and analysis of a system with two cooperative manipulators working together on a common task. The task is defined as the transportation of an object in space. The cooperative system is the dual-arm of the humanoid robot Nao, where the serial architecture of each arm has five degrees of freedom. The kinematics representing the closed chain system is studied. The mobility of the closed-loop system is analyzed and the nature of the possible motions explored. The stiffness of some motors can be reduced until they behave as passive joints. Certain joints are then chosen as actuated (independent) and the others as passive (dependent). The serial and parallel singular configurations of the system are considered. From the kinematic analysis, admissible and inadmissible minimum actuation schemes are analyzed. Furthermore the dynamic performance of the schemes is compared to find the optimum minimum actuation scheme.
\end{abstract}

\section{Introduction}

The use of dual independent arms for manipulation tasks has several advantages over classical serial robots. For instance, a cooperative system can reduce the need for custom fixtures,

*Corresponding author: Stéphane Caro; Email: stephane.caro@irccyn.ec-nantes.fr; Tel: +33 24037 69 68; Fax: +33240376930 
permit the use of a simpler tool and handle flexible objects. Furthermore heavy objects can be manipulated by sharing the load among many robots. On the other hand, the use of dual independent arms requires more advanced modeling and control techniques.

When grasping a common object, cooperative manipulators form a closed chain system. One approach to model such a system is to formulate kinematic relations that create a task space. The main methods are known as Symmetric formulation [1] and Task orientated formulation [2, 3]. For a grasped object, a cooperative task space is created that consists of twelve velocity variables. The velocity variables describe both the object motion in space and the relative motion between the end-effectors. The latter can be seen as a method to control the internal forces on the object. The system can also be viewed as a redundantly actuated parallel manipulator. In this case, kinematic constraint equations are derived that establish a relationship between the chosen actuated and passive joint variables $[4,5,6,7,8]$. In order to satisfy the close chain constraints, the passive joints must adopt values that ensure loop closure throughout the trajectory.

A lower mobility robotic manipulator is a system that has less than 6 degrees of freedom. Unlike full mobility systems, lower mobility systems suffer from three types of singularities, limb (serial) singularities, actuation and constraint (parallel) singularities [9]. For a cooperative manipulator system the presence of parallel singularities is explored in [5]. A study regarding the valid selection of actuators is carried in [7]. In both cases an analysis of the Jacobian matrix of the robot is required. Alternatively, the geometric tool known as screw theory can be used to locate and understand singularities in closed chain mechanisms [10].

In this paper the cooperative system, defined by the two arms of Aldebaran NAO T14 humanoid robot and a grasped object, is examined. The system has been modeled as a closed chain mechanism (Section 2). Many industrial applications using multiple robots can be modeled as one closed chain system, mainly tasks requiring internal force control of a target object. For instance, the ARMS project ${ }^{1}$ aims to contribute to the increasing mechanization of the meat industry. In this scenario a multi-arm system is designed to process meat. One arm is used to cut the muscle while the second pulls and tears in order to perform the separation. The closed chain is completed by a deformable object, i.e. an object of multi DOF representing the meat. The robot configuration, object DOF and task specification drive the choice of independent actuators. The passive or dependent actuators are then adapted to fulfill constraint equations. The advantage of using minimum actuators is twofold. Firstly they lead to a simpler control scheme, since there are less variables to control. Secondly by using passive joints, antagonistic

\footnotetext{
${ }^{1}$ http://arms.irccyn.ec-nantes.fr/
} 
forces on the object due to poor trajectory tracking can be avoided.

In this paper we make use of screw theory to analyze the system's mobility, singularities and motion type. The benefit of this approach is that special configurations such as the loss of stiffness, loss of DOF etc., can be determined without the complex derivation of the Jacobian matrices (or their inverses) (Sections 3 and 6). From this analysis all possible minimum actuation schemes are examined and enumerated for the two cooperative manipulators. The inadmissible actuation schemes, the reasons behind and the effects of this inadmissibility, are illustrated and explained in geometrical terms. Furthermore the analysis of the constraint screws give a greater insight into the reasons for these singular configurations and allows us to discover the link between the actuation scheme and the system performance. Finally the dynamic performance is considered. In Section 8 the performance of each actuation scheme is assessed with respect to power loss and maximum torque for a large number of trajectories. These methods permit the selection of an optimum actuation scheme.

\section{System Description}

The dual-arm system analyzed in this paper is shown in Fig. 1, while the kinematic architecture of the arms are given in Fig. 2. Each arm of the robot has five independent revolute joints. The right arm consists of joints 1-5 and the left arm consists of joints 6-10.

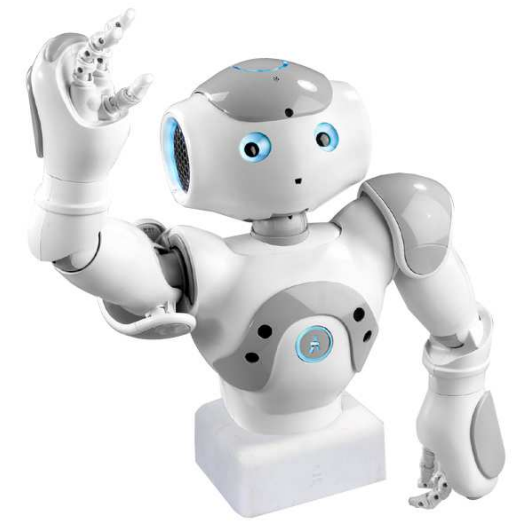

Figure 1: Nao T14, (Courtesy of Aldebaran Robotics)

The robotic system is described by the Modified Denavit-Hartenberg (MDH) notation as proposed by Khalil and Kleinfiger [11], given in Table 1 and modeled using [12]. The transformation matrix ${ }^{a(j)} \mathbf{T}_{j}$, from frame $a(j)$, the antecedent of $j$, to frame $j$ is the $4 \times 4$ matrix given 


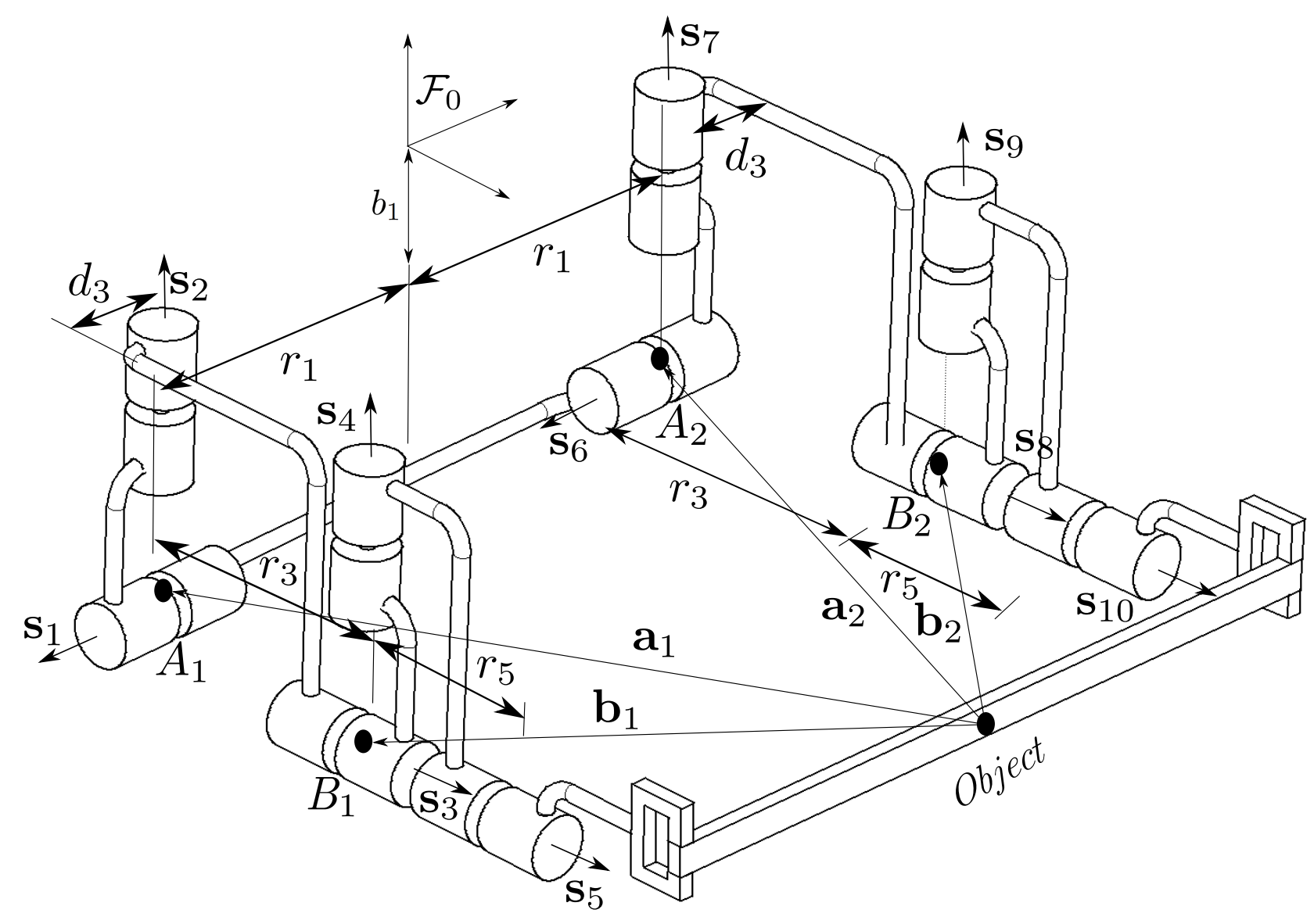

Figure 2: Closed-Loop Formulation

by:

$$
\begin{array}{r}
{ }^{a(j)} \mathbf{T}_{j}=\operatorname{rot}_{z}\left(\gamma_{j}\right) \cdot \operatorname{trans}_{z}\left(b_{j}\right) \\
\cdot \operatorname{rot}_{x}\left(\alpha_{j}\right) \cdot \operatorname{trans}_{x}\left(d_{j}\right) \\
\cdot \operatorname{rot}_{z j}\left(\theta_{j}\right) \cdot \operatorname{trans}_{z}\left(r_{j}\right)
\end{array}
$$

where $\operatorname{rot}_{i}(\theta)$ indicates a rotation of $\theta$ about the direction of the $i$ th axis and $\operatorname{trans}_{i}(l)$ a translation of $l$ meters along the $i$ th axis. It should be noted that $\gamma_{j}=b_{j}=0$ when $\mathbf{x}_{(a(j))}$ is perpendicular to $\mathbf{z}_{j} . q_{i}$ denotes the joint variable $i$ and is equal to $\theta_{i}$.

Once the object is grasped, a closed-loop is formed, as shown in Fig. 2. The system has, in this case, only nine bodies. Joint 10 is chosen as the cut joint therefore, to define the equivalent tree structure, link 5 of the closed chain now contains the object, link 5 and link 10. Frame 10 becomes fixed on link 5. We introduce frame 11, which is aligned to frame 10, but its antecedent is frame 5 . The parameters of frame 11 are defined once the robot has grasped the object. The locations of frame 10 and frame 11 are equivalent when calculated via 
Table 1: MDH Parameters of the closed-loop chain

\begin{tabular}{cccccccc}
\hline \hline$j$ & $a(j)$ & $\gamma$ & $b$ & $d$ & $\alpha$ & $\theta$ & $r$ \\
\hline 1 & 0 & 0 & $b_{1}$ & 0 & $-\pi / 2$ & $\theta_{1}$ & -0.098 \\
2 & 1 & 0 & 0 & 0 & $\pi / 2$ & $\theta_{2}$ & 0 \\
3 & 2 & 0 & 0 & -0.015 & $\pi / 2$ & $\theta_{3}$ & 0.105 \\
4 & 3 & 0 & 0 & 0 & $-\pi / 2$ & $\theta_{4}$ & 0 \\
5 & 4 & 0 & 0 & 0 & $\pi / 2$ & $\theta_{5}$ & 0.05595 \\
6 & 0 & 0 & $b_{1}$ & 0 & $-\pi / 2$ & $\theta_{6}$ & 0.098 \\
7 & 6 & 0 & 0 & 0 & $\pi / 2$ & $\theta_{7}$ & 0 \\
8 & 7 & 0 & 0 & 0.015 & $\pi / 2$ & $\theta_{8}$ & 0.105 \\
9 & 8 & 0 & 0 & 0 & $-\pi / 2$ & $\theta_{9}$ & 0 \\
10 & 9 & 0 & 0 & 0 & $\pi / 2$ & $\theta_{10}$ & 0.05595 \\
11 & 5 & $\gamma_{11}$ & $b_{11}$ & $d_{11}$ & $\alpha_{11}$ & $\theta_{11}$ & $r_{11}$ \\
\hline \hline
\end{tabular}

either chain. This ensures a constant object grasp throughout the trajectory. The geometric constraint equations are given by:

$$
{ }^{0} \mathbf{T}_{1}{ }^{1} \mathbf{T}_{2}{ }^{2} \mathbf{T}_{3}{ }^{3} \mathbf{T}_{4}{ }^{4} \mathbf{T}_{5}{ }^{5} \mathbf{T}_{11}={ }^{0} \mathbf{T}_{6}{ }^{6} \mathbf{T}_{7}{ }^{7} \mathbf{T}_{8}{ }^{8} \mathbf{T}_{9}{ }^{9} \mathbf{T}_{10}
$$

It should be noted that since the axes of joints 1 and 2 intersect at point $A_{1}$, while the axes of joints 3,4 and 5 intersect at point $B_{1}$, the arm of the robot can be represented as a U-joint and a spherical joint serially connected. Therefore in the closed-loop configuration the robot is viewed as a 2-US parallel architecture.

The kinematic constraints are given by:

$$
\left[\begin{array}{c}
{ }^{0} \omega_{11} \\
{ }^{0} \mathbf{v}_{11}
\end{array}\right]=\left[\begin{array}{c}
{ }^{0} \omega_{10} \\
{ }^{0} \mathbf{v}_{10}
\end{array}\right]=\left[\begin{array}{c}
0 \omega_{o b j} \\
{ }^{0} \mathbf{v}_{o b j}
\end{array}\right]
$$




$$
\begin{gathered}
{\left[\begin{array}{c}
0 \\
{ }^{0} \omega_{o b j} \\
{ }^{0} \mathbf{v}_{o b j}
\end{array}\right]={ }^{0} \mathbf{J}_{11}\left[\begin{array}{c}
\dot{q}_{1} \\
\dot{q}_{2} \\
\dot{q}_{3} \\
\dot{q}_{4} \\
\dot{q}_{5}
\end{array}\right]={ }^{0} \mathbf{J}_{11} \dot{\mathbf{q}}_{r}} \\
{\left[\begin{array}{c}
{ }^{0} \omega_{o b j} \\
{ }^{0} \mathbf{v}_{o b j}
\end{array}\right]={ }^{0} \mathbf{J}_{10}\left[\begin{array}{c}
\dot{q}_{6} \\
\dot{q}_{7} \\
\dot{q}_{8} \\
\dot{q}_{9} \\
\dot{q}_{10}
\end{array}\right]={ }^{0} \mathbf{J}_{10} \dot{\mathbf{q}}_{l}}
\end{gathered}
$$

The superscript ${ }^{0}$ indicates that the variable is represented in the fixed world frame $\mathcal{F}_{0}$. As frames 10 and 11 are the same, from (5) and (4):

$$
{ }^{0} \mathbf{J}_{11} \dot{\mathbf{q}}_{r}={ }^{0} \mathbf{J}_{10} \dot{\mathbf{q}}_{l}
$$

or rewritten as

$$
\mathbf{J}_{s}\left[\begin{array}{c}
\dot{\mathbf{q}}_{r} \\
\dot{\mathbf{q}}_{l}
\end{array}\right]=0 \quad \text { where } \quad \mathbf{J}_{s}=\left[\begin{array}{ll}
{ }^{0} \mathbf{J}_{11} & -{ }^{0} \mathbf{J}_{10}
\end{array}\right]
$$

where $\dot{\mathbf{q}}_{r}$ and $\dot{\mathbf{q}}_{l}$ contain the joint velocities of the right arm and the left arm, respectively. ${ }^{0} \mathbf{v}_{j}$ is the linear velocity and ${ }^{0} \omega_{j}$ the angular velocity of frame $j$ with respect to frame $0,{ }^{0} \mathbf{J}_{j}$ is the $6 \times 5$ kinematic Jacobian matrix of frame $j$ with respect to frame 0 . By rearranging the rows and columns of (6), a relationship is obtained between the passive joint velocities and the actuated joint velocities:

$$
\left[\begin{array}{ccc}
\mathbf{G}_{a} & \mathbf{G}_{p} & \mathbf{0} \\
\mathbf{G}_{a c} & \mathbf{G}_{p c} & \mathbf{G}_{c}
\end{array}\right]\left[\begin{array}{c}
\dot{\mathbf{q}}_{a} \\
\dot{\mathbf{q}}_{p} \\
\dot{\mathbf{q}}_{c}
\end{array}\right]=\mathbf{0}
$$

$\mathbf{q}_{a}, \mathbf{q}_{p}$ and $\mathbf{q}_{c}$ denote the vectors containing the actuated, passive and cut joints respectively. Upon differentiation of (8) with respect to time the acceleration constraints equation is ex- 
pressed as:

$$
\left[\begin{array}{ccc}
\mathbf{G}_{a} & \mathbf{G}_{p} & \mathbf{0} \\
\mathbf{G}_{a c} & \mathbf{G}_{p c} & \mathbf{G}_{c}
\end{array}\right]\left[\begin{array}{c}
\ddot{\mathbf{q}}_{a} \\
\ddot{\mathbf{q}}_{p} \\
\ddot{\mathbf{q}}_{c}
\end{array}\right]+\dot{\mathbf{J}}_{s} \dot{\mathbf{q}}=0
$$

From (8), we obtain:

$$
\begin{gathered}
\dot{\mathbf{q}}_{p}=-\mathbf{G}_{p}^{-1} \mathbf{G}_{a} \dot{\mathbf{q}}_{a} \\
\dot{\mathbf{q}}_{c}=-\mathbf{G}_{c}^{-1}\left(\mathbf{G}_{a c}-\mathbf{G}_{p c} \mathbf{G}_{p}^{-1} \mathbf{G}_{a}\right) \dot{\mathbf{q}}_{a}
\end{gathered}
$$

Furthermore from (3), (4) and (5):

$$
\left[\begin{array}{ll}
\frac{1}{2}{ }^{0} \mathbf{J}_{10} & \frac{1}{2}^{0} \mathbf{J}_{11}
\end{array}\right]\left[\begin{array}{c}
\dot{\mathbf{q}}_{r} \\
\dot{\mathbf{q}}_{l}
\end{array}\right]=\left[\begin{array}{c}
{ }^{0} \omega_{o b j} \\
{ }^{0} \mathbf{v}_{o b j}
\end{array}\right]
$$

By rearranging the rows and columns of (12), a relationship is obtained between the passive joint velocities, the actuated joint velocities and the object twist can be found:

$$
\left[\begin{array}{lll}
\mathbf{J}_{a} & \mathbf{J}_{p} & \mathbf{J}_{c}
\end{array}\right]\left[\begin{array}{c}
\dot{\mathbf{q}}_{a} \\
\dot{\mathbf{q}}_{p} \\
\dot{\mathbf{q}}_{c}
\end{array}\right]=\left[\begin{array}{c}
{ }^{0} \omega_{o b j} \\
{ }^{0} \mathbf{v}_{o b j}
\end{array}\right]
$$

Finally an actuated Jacobian matrix, $\mathbf{J}_{a c t}$ is derived that defines a relationship between the actuated joint velocities and the object velocity. Using (13) and (8):

$$
\begin{aligned}
\mathbf{J}_{a c t} \dot{\mathbf{q}}_{a} & =\left[\begin{array}{c}
{ }^{0} \omega_{o b j} \\
{ }^{0} \mathbf{v}_{o b j}
\end{array}\right] \\
\mathbf{J}_{a c t} & =\mathbf{J}_{a}+\mathbf{J}_{p}\left(-\mathbf{G}_{p}^{-1} \mathbf{G}_{a}\right) \\
& +\mathbf{J}_{c}\left(-\mathbf{G}_{c}^{-1}\left(\mathbf{G}_{a c}-\mathbf{G}_{p c} \mathbf{G}_{p}^{-1} \mathbf{G}_{a}\right)\right)
\end{aligned}
$$

The mobility of the system, given in section 3 , is equal to 4 . Hence the dimension of $\mathbf{G}_{a}$ is $5 \times 4, \mathbf{G}_{p}$ is $5 \times 5, \mathbf{G}_{c}$ is a scalar that is, due to the modeling procedure, always equal to one, where 4,5 and 1 are the numbers of active, passive and cut joints, respectively. $\mathbf{G}_{p}$ degenerates at configurations where the constraints become linearly dependent, as shown in Section 7. 


\section{Mobility Analysis}

The degree of freedom (DOF) of the system is equal to the number of independent coordinates required to control it. The DOF can be obtained by several methods for example ChebychevGrübler-Kutzbach, or Gogu's Method [13]. In order to elucidate the motion type, screw theory is used [14]. In summary, each serial arm has 5-DOF however once the object is firmly grasped by the two arms a closed chain is formed and the object DOF becomes four. In the following sections, screw theory is recalled and applied to the closed-loop system. Firstly the twists associated with each arm are defined. Then the wrenches applied on the object are obtained. Finally, using this information the DOF of the object can be analyzed.

The screw theory analysis is carried out with respect to an intermediate frame $\hat{\mathcal{F}}_{o b j}$ positioned on the object. More precisely, this frame is described as the frame whose origin coincides with the origin of the object frame $\mathcal{F}_{o b j}$, but whose orientation is always equal to that of the world frame $\mathcal{F}_{0}$. An illustration is given in Fig. 3.

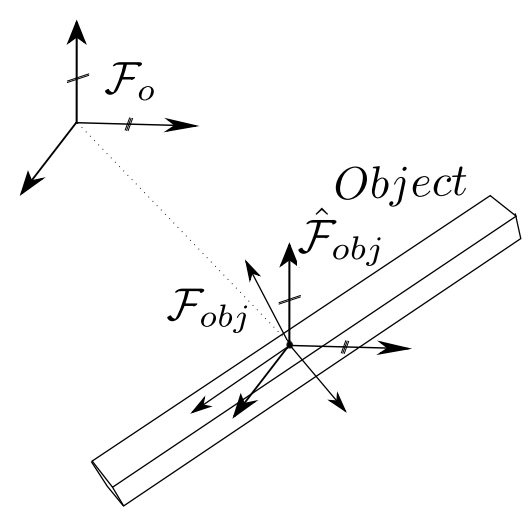

Figure 3: Representation of origin frame

\subsection{Screw Theory}

Screw theory can be used to analyze the instantaneous motions of complex mechanisms [15, $16,17]$. A screw of pitch $\lambda$ is defined as:

$$
\$_{\lambda}=\left[\begin{array}{c}
\mathbf{s} \\
\mathbf{s} \times \mathbf{r}+\lambda \mathbf{s}
\end{array}\right]
$$

s a unit vector along the axis of the screw. $\mathbf{r}$ is a vector directed from any point on the axis of the screw to the origin $\mathcal{F}_{o}$. A zero-pitch screw and an infinite-pitch screw are expressed 
respectively as follows:

$$
\begin{aligned}
& \$_{0}=\left[\begin{array}{c}
\mathbf{s} \\
\mathbf{s} \times \mathbf{r}
\end{array}\right] \\
& \$_{\infty}=\left[\begin{array}{c}
\mathbf{0}_{3 \times 1} \\
\mathbf{s}
\end{array}\right]
\end{aligned}
$$

For every screw system, consisting of $n$ linearly independent screws, there exists a reciprocal screw system of dimension $6-n$. Two screws $\$_{1}$ and $\$_{2}$ are reciprocal if their instantaneous power is zero, namely,

$$
\left(\left[\begin{array}{cc}
\mathbf{0}_{3 \times 3} & \mathbf{I}_{3} \\
\mathbf{I}_{3} & \mathbf{0}_{3 \times 3}
\end{array}\right] \$_{1}\right)^{T} \$_{2}=0
$$

The following reciprocity conditions are defined in [17]:

1. $\$_{0}$ is reciprocal to $\$_{\infty}$ if and only if their axes are orthogonal;

2. $\$_{\infty}$ is always reciprocal to another $\$_{\infty}$;

3. Two $\$_{0}$ are reciprocal if and only if their axes are coplanar (two coplanar axes are either intersecting or parallel).

A zero-pitch twist $\nu_{0}$ corresponds to a pure rotation about its axis. An $\infty$-pitch twist $\nu_{\infty}$ corresponds to a pure translation along its direction. A zero-pitch wrench $\zeta_{0}$ corresponds to a pure force along its axis. An $\infty$-pitch wrench $\zeta_{\infty}$ corresponds to a pure moment about its direction.

\subsection{Twist System of Nao Robot}

The twist system associated with one arm of the Nao robot is spanned by five zero-pitch twists. The twist system, $\mathrm{T}^{\mathrm{r}}$, of the right arm is spanned by $\nu_{01}, \nu_{02}, \nu_{03}, \nu_{04}, \nu_{05}$, :

$$
\nu_{0 i}=\left[\begin{array}{c}
\mathbf{s}_{i} \\
\mathbf{a}_{\mathbf{1}} \times \mathbf{s}_{i}
\end{array}\right] \quad(20 \mathrm{a}) \quad \nu_{0 j}=\left[\begin{array}{c}
\mathbf{s}_{j} \\
\mathbf{b}_{\mathbf{1}} \times \mathbf{s}_{j}
\end{array}\right]
$$

where $i=1,2$ and $j=3,4,5$.

Likewise, the twist system of the left arm, $\mathrm{T}^{1}$, is spanned by $\nu_{06}, \nu_{07}, \nu_{08}, \nu_{09}, \nu_{010}$,

$$
\nu_{0 i}=\left[\begin{array}{c}
\mathbf{s}_{i} \\
\mathbf{a}_{2} \times \mathbf{s}_{i}
\end{array}\right] \quad(21 \mathrm{a}) \quad \nu_{0 j}=\left[\begin{array}{c}
\mathbf{s}_{j} \\
\mathbf{b}_{2} \times \mathbf{s}_{j}
\end{array}\right]
$$


where $i=6,7$ and $j=8,9,10$.

$\mathbf{s}_{1}, \mathbf{s}_{2}$ are the unit vectors of the first and second revolute joint axes of the U-joint of the right arm, while $\mathbf{s}_{6}, \mathbf{s}_{7}$ are the equivalent unit vectors of the left arm. $\mathbf{s}_{3}, \mathbf{s}_{4}$ and $\mathbf{s}_{5}$ are the unit vectors of the revolute joints associated with the S-joint of the right arm, while $\mathbf{s}_{8}, \mathbf{s}_{9}$ and $\mathbf{s}_{10}$ are the equivalent unit vectors of the left arm. Let $\mathbf{a}_{1}, \mathbf{a}_{2}, \mathbf{b}_{1}$ and $\mathbf{b}_{2}$ represent the Cartesian vectors from $A_{1}, A_{2}, B_{1}$ and $B_{2}$ to the origin $\hat{\mathcal{F}}_{o b j}$ respectively, as shown in Fig. 2. The twist system $\mathrm{T}$ of the Nao robot is the intersection of $\mathrm{T}^{r}$ and $\mathrm{T}^{l}$.

\subsection{Constraint Wrench System of Nao Robot}

From Section 3.2, the twist systems $\mathrm{T}^{r}$ and $\mathrm{T}^{l}$ associated with the right and left arms of Nao robot are 5-systems when the arms are not in a singular configuration. Therefore, outside singular configurations, the constraint wrench system $\mathrm{W}_{c}^{r}$ of the right arm and the constraint wrench system $\mathrm{W}_{c}^{l}$ of the left arm are $(6-5)$-systems, i.e., 1-systems. $\mathrm{W}_{c}^{r}$ and $\mathrm{W}_{c}^{l}$ are reciprocal to $\mathrm{T}^{r}$ and $\mathrm{T}^{l}$ respectively and are expressed as follows:

$$
\begin{aligned}
\mathrm{W}_{c}^{r} & =\operatorname{span}\left(\zeta_{0 r}^{c}\right) \\
\mathrm{W}_{c}^{l} & =\operatorname{span}\left(\zeta_{0 l}^{c}\right)
\end{aligned}
$$

From reciprocity condition (3):

$$
\begin{aligned}
& \zeta_{0 r}^{c}=\left[\begin{array}{c}
\mathbf{u}_{r} \\
\mathbf{b}_{1} \times \mathbf{u}_{r}
\end{array}\right] \\
& \zeta_{0 l}^{c}=\left[\begin{array}{c}
\mathbf{u}_{l} \\
\mathbf{b}_{2} \times \mathbf{u}_{l}
\end{array}\right]
\end{aligned}
$$

$\mathbf{u}_{r}$ is the unit vector intersecting points $A_{1}$ and $B_{1}$, while $\mathbf{u}_{l}$ is the unit vector intersecting points $A_{2}$ and $B_{2}$. The constraint wrench system $\mathrm{W}^{c}$ of the rigid object firmly grasped by the hands of the robot is the linear combination of the wrench systems $\mathrm{W}_{c}^{r}$ and $\mathrm{W}_{c}^{l}$, namely,

$$
\mathrm{W}^{c}=\mathrm{W}_{c}^{r}+\mathrm{W}_{c}^{l}=\operatorname{span}\left(\zeta_{0 r}^{c}, \zeta_{0 l}^{c}\right)
$$

As a result, the constraint wrench system $\mathrm{W}^{c}$ of Nao robot is a 2-system spanned by two pure forces (zero pitch wrenches) as long as the robot does not reach a constraint singularity. These forces intersect both the U-joint and the S-joint of the arms, i.e., the axis of the twists of each arm. 


\section{Actuation Schemes}

In this section a selection criterion is given for suitable actuated joints using the actuator wrenches described in Section 3.1. By focusing on the minimum number of actuators required to fully control the 4-DOF of the object, it is possible to find $\left(\frac{10 !}{4 !(10-4) !}=\right) 210$ possible actuation schemes. $\mathbf{q}_{a}$ denotes the vector of actuation joints. $\mathbf{q}_{a}=\left[\begin{array}{llll}q_{i} & q_{j} & q_{k} & q_{l}\end{array}\right]$ means that the $i^{t h}, j^{t h}, k^{t h}$ and $l^{t h}$ joints of the closed loop kinematic chain are actuated where $i, j, k, l=$ $1 \ldots 10, i \neq j \neq k \neq l$.

Due to the symmetry of the two arms, the number of kinematically distinct schemes is significantly less than 210. For example we treat the actuation scheme no. $1 \mathbf{q}_{a}=\left[\begin{array}{llll}q_{1} & q_{2} & q_{3} & q_{4}\end{array}\right]$ and its mirror image $\mathbf{q}_{a}=\left[\begin{array}{llll}q_{6} & q_{7} & q_{8} & q_{9}\end{array}\right]$ as equal. On the other hand scheme no.23 $\mathbf{q}_{a}=$ $\left[\begin{array}{llll}q_{1} & q_{2} & q_{6} & q_{7}\end{array}\right]$ has no symmetric equivalent. By excluding symmetrical actuation schemes, 110 actuation schemes remain as given in Table 2. Each scheme has its actuation scheme number written on the left. Schemes that have no symmetric equivalent are marked with the superscript ${ }^{*}$.

The schemes can be subdivided into either inadmissible actuation schemes or admissible actuation schemes. The reason for the inadmissibility, a degeneracy in the global wrench system, is demonstrated in this section. The inadmissible schemes, 39 in total, are written with a strikethrough notation. The closed-loop scheme contains ten revolute joints, each passive joint is denoted as 0 whereas each actuated joint is denoted as 1 . For example scheme no. 4, $\mathbf{q}_{a}=\left[\begin{array}{llll}q_{1} & q_{2} & q_{3} & q_{8}\end{array}\right]$ is represented as 11100-00100.

In summary, from the 210 schemes, by excluding all the schemes which are either inadmissible or have symmetric equivalent, the total number of admissible kinematically distinct actuation schemes is found to be 71 . Furthermore, schemes 1, 7, 13, 34 and 77, though technically feasible, create systems where one arm contains only passive joints. Therefore if excluded the number of valid cooperative schemes would reduce to 67 .

\subsection{Actuation System of Nao Robot arms}

In order to analyze the actuation schemes, the wrenches exerted by each joint when chosen as an actuated joint are examined. The wrench exerted by an actuator on the object is defined as the wrench reciprocal to all the twists of the specified arm except the twist corresponding to the selected actuator itself. The actuator wrench for joint $j$ is denoted as $\zeta_{\lambda j}^{a}$. The zero pitch actuation wrenches associated with the right arm are deduced from the reciprocity conditions as given in Section 3.1 with $i=1,2$ and $j=3,4,5$ : 


$$
\zeta_{0 i}^{a}=\left[\begin{array}{c}
\mathbf{u}_{i} \\
\mathbf{b}_{1} \times \mathbf{u}_{i}
\end{array}\right]
$$

$$
\zeta_{0 j}^{a}=\left[\begin{array}{c}
\mathbf{u}_{j} \\
\mathbf{a}_{1} \times \mathbf{u}_{j}
\end{array}\right]
$$

$\mathbf{u}_{1}$ is the unit vector passing through point $B_{1}$ (thus reciprocal to twists $\nu_{03}, \nu_{04}$ and $\nu_{05}$ ) and parallel to $\mathbf{s}_{2}$ (thus reciprocal to twist $\nu_{02}$ ). $\mathbf{u}_{2}$ is the unit vector passing through point $B_{1}$ and parallel to $\mathbf{s}_{1}$ (thus reciprocal to joints $\nu_{01}$ ). $\mathbf{u}_{3}$ is the unit vector of the intersection line $\mathcal{L}_{3}$ of planes $\mathcal{P}_{45}$ and $\mathcal{P}_{12} . \mathbf{u}_{4}$ is the unit vector of the intersection line $\mathcal{L}_{4}$ of planes $\mathcal{P}_{35}$ and $\mathcal{P}_{12}$. $\mathbf{u}_{5}$ is the unit vector of the intersection line $\mathcal{L}_{5}$ of planes $\mathcal{P}_{34}$ and $\mathcal{P}_{12}$, where $\mathcal{P}_{12}$ is the plane spanned by vectors $\mathbf{s}_{1}$ and $\mathbf{s}_{2}$ passing through point $A_{1} . \mathcal{P}_{34}$ is the plane spanned by vectors $\mathbf{s}_{3}$ and $\mathbf{s}_{4}$ passing through point $B_{1} . \mathcal{P}_{35}$ is the plane spanned by vectors $\mathbf{s}_{3}$ and $\mathbf{s}_{5}$ passing through point $B_{1} . \mathcal{P}_{45}$ is the plane spanned by vectors $\mathbf{s}_{4}$ and $\mathbf{s}_{5}$ passing through point $B_{1}$.

Similarly, the zero pitch actuation wrenches associated with the left arm are defined as:

$$
\zeta_{0 i}^{a}=\left[\begin{array}{c}
\mathbf{u}_{i} \\
\mathbf{b}_{2} \times \mathbf{u}_{i}
\end{array}\right] \quad(28 \mathrm{a}) \quad \zeta_{0 j}^{a}=\left[\begin{array}{c}
\mathbf{u}_{j} \\
\mathbf{a}_{2} \times \mathbf{u}_{j}
\end{array}\right]
$$

where $i=6,7$ and $j=8,9,10$.

$\mathbf{u}_{6}$ is the unit vector passing through point $B_{2}$ (thus reciprocal to twists $\nu_{08}, \nu_{09}$ and $\nu_{010}$ ) and parallel to $\mathbf{s}_{7}$ (thus reciprocal to joints $\nu_{0} 7$ ). $\mathbf{u}_{7}$ is the unit vector passing through point $B_{2}$ and parallel to $\mathbf{s}_{6}$ (thus reciprocal to twist $\nu_{06}$ ). $\mathbf{u}_{8}$ is the unit vector of the intersection line $\mathcal{L}_{8}$ of planes $\mathcal{P}_{910}$ and $\mathcal{P}_{67} . \quad \mathbf{u}_{9}$ is the unit vector of the intersection line $\mathcal{L}_{9}$ of planes $\mathcal{P}_{810}$ and $\mathcal{P}_{67}$. $\mathbf{u}_{10}$ is the unit vector of the intersection line $\mathcal{L}_{10}$ of planes $\mathcal{P}_{89}$ and $\mathcal{P}_{67}$, where $\mathcal{P}_{67}$ is the plane spanned by vectors $\mathbf{s}_{6}$ and $\mathbf{s}_{7}$ passing through point $A_{2} . \mathcal{P}_{89}$ is the plane spanned by vectors $\mathbf{s}_{8}$ and $\mathbf{s}_{9}$ passing through point $B_{2} . \mathcal{P}_{810}$ is the plane spanned by vectors $\mathbf{s}_{8}$ and $\mathbf{s}_{10}$ passing through point $B_{2} . \mathcal{P}_{910}$ is the plane spanned by vectors $\mathbf{s}_{9}$ and $\mathbf{s}_{10}$ passing through point $B_{2}$. For clarity, Fig. 4 shows the unit vectors associated with the wrench system of the right arm.

For any choice of actuators, the actuator wrench system is spanned by the chosen actuator wrenches. Taking for example, scheme no. $1 \mathbf{q}_{a}=\left[\begin{array}{llll}q_{1} & q_{2} & q_{3} & q_{4}\end{array}\right]$ (11110-00000). The actuation wrench system, $\mathrm{W}^{a}$, is spanned by the following four zero pitch wrenches:

$$
\mathrm{W}^{a}=\operatorname{span}\left(\zeta_{01}^{a}, \quad \zeta_{02}^{a}, \quad \zeta_{03}^{a}, \quad \zeta_{04}^{a}\right)
$$

The global wrench system $\mathrm{W}$ is the wrench system spanned by the constraint wrench system $\mathrm{W}^{c}$ 


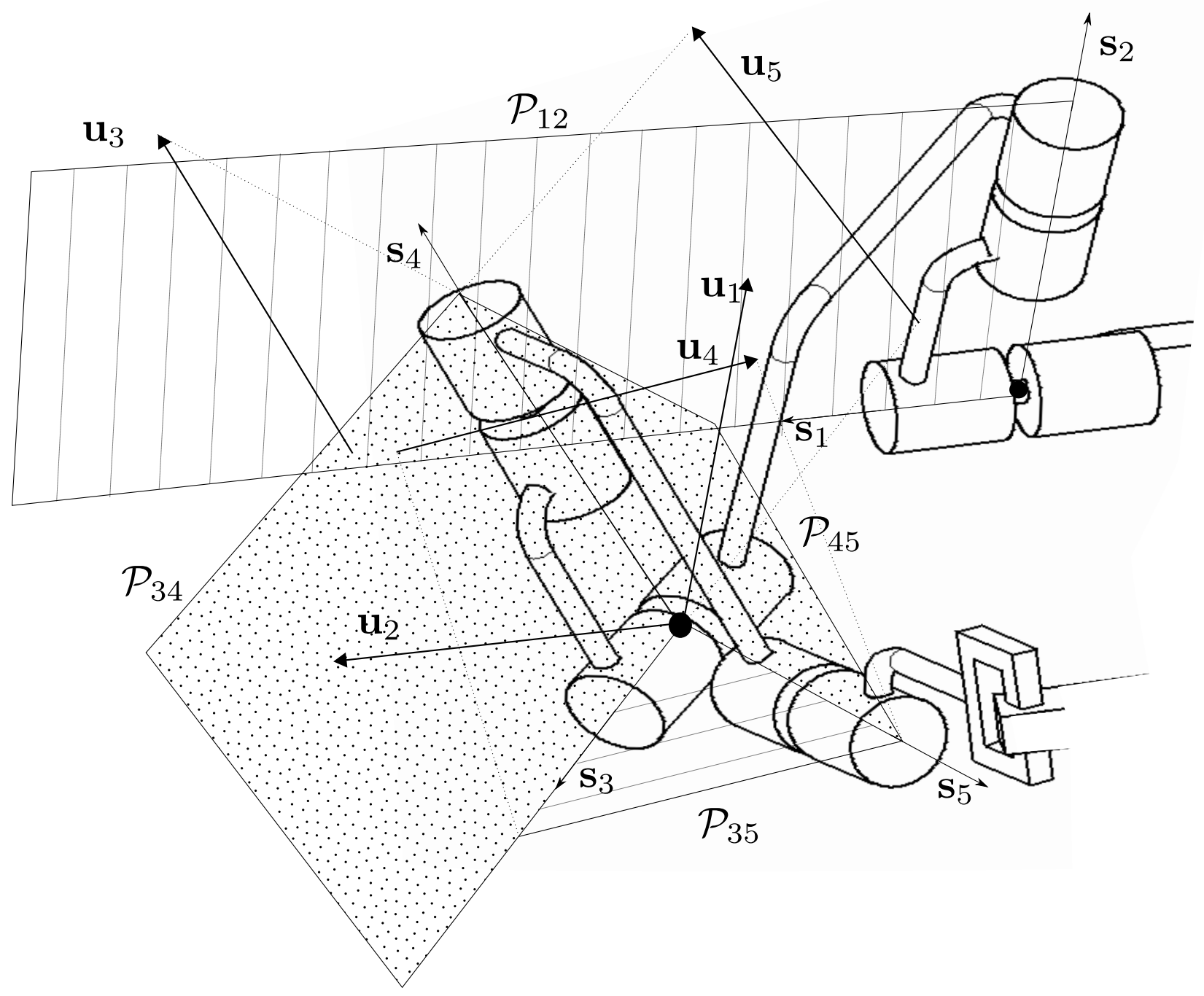

Figure 4: Unit vectors of wrench system of right arm

and the actuation wrench system $\mathrm{w}^{a}$, namely:

$$
\mathrm{W}=\operatorname{span}\left(\mathrm{W}^{c}, \mathrm{~W}^{a}\right)
$$

For this example scheme:

$$
\mathrm{W}=\operatorname{span}\left(\zeta_{0 r}^{c}, \quad \zeta_{0 l}^{c}, \quad \zeta_{01}^{a}, \quad \zeta_{02}^{a}, \quad \zeta_{03}^{a}, \quad \zeta_{04}^{a}\right)
$$


Table 2: All actuation schemes, ${ }^{*}$ indicates that there is no symetric equivalent

\begin{tabular}{|c|c|c|c|c|c|c|c|}
\hline 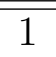 & $111110-00000$ & 29 & 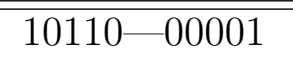 & "57 & "10011-00001 & 85 & $01101-00010$ \\
\hline 2 & $11100-00001$ & 30 & $10110-00010$ & 58 & $10011-00010$ & 86 & $\theta 1101-00100$ \\
\hline 3 & $11100-00010$ & 31 & $10110-00100$ & 59 & $10011-00100$ & 87 & $01101-01000$ \\
\hline 4 & $11100-00100$ & 32 & $10-01000$ & 60 & -0 & 88 & $\theta 1010-\theta 0011$ \\
\hline 5 & $11100-01000$ & 33 & $10110-10000$ & 61 & $10011-10000$ & 89 & $\theta 1010-\theta 0101$ \\
\hline 6 & $11100-10000$ & 34 & $10111-00000$ & 62 & $10000-00111$ & 90 & $\theta 101 \theta-\theta 011 \theta$ \\
\hline 7 & $11101-00000$ & 35 & $10100-00011$ & 63 & $10000-01011$ & 91 & $01010-01001$ \\
\hline 8 & $11010-00001$ & 36 & $10100-00101$ & 64 & $10000-01101$ & 92 & $01010-01010^{*}$ \\
\hline 9 & $11010-00010$ & 37 & $10100-00110$ & 65 & $00-0$ & 93 & $01011-00001$ \\
\hline 10 & $11010-00100$ & 38 & $10100-01001$ & 66 & $01-00011$ & 94 & $\theta 1011-\theta 001 \theta$ \\
\hline 11 & $11010-01000$ & 39 & $10100-01010$ & 67 & $10001-00101$ & 95 & $\theta 1011-\theta 010 \theta$ \\
\hline 12 & $11010-10000$ & 40 & $10100-01100$ & 68 & $10001-00$ & 96 & $\theta 1011-\theta 100 \theta$ \\
\hline 13 & $11011-00000$ & 41 & $10100-10001$ & 69 & $10001-01001$ & 97 & $01000-00111$ \\
\hline 14 & $11000-00011$ & 42 & $10100-10010$ & 70 & $10001-01$ & 98 & $01001-00011$ \\
\hline 15 & 11000 & 43 & $10100-10100^{*}$ & 71 & 00 & 99 & $\theta 1001-\theta t$ \\
\hline 16 & $11000-00110$ & 44 & $10101-00001$ & 72 & $10001-10001^{*}$ & 100 & $\theta 1001-00110$ \\
\hline 17 & $11000-01001$ & 45 & $10101-00010$ & 73 & $\theta 111 \theta-\theta+\theta \theta 1$ & 101 & $\theta 1001-\theta 1001^{*}$ \\
\hline 18 & $11000-01010$ & 46 & $10101-00100$ & 74 & $\theta 111 \theta-\theta 001 \theta$ & 102 & $\theta 011 \theta-\theta 0011$ \\
\hline 19 & $11000-01100$ & 47 & $10101-01000$ & 75 & $01110-00100$ & 103 & $00110-00101$ \\
\hline 20 & $11000-10001$ & 48 & $10101-10000$ & 76 & $01110-01000$ & 104 & $\theta 0110-00110^{*}$ \\
\hline 21 & $11000-10010$ & 49 & $10010-00011$ & 77 & $\theta 1111-\theta 0000$ & 105 & $00111-00001$ \\
\hline 22 & $11000-10100$ & 50 & $10010-00101$ & 78 & $01100-00011$ & 106 & $00111-00010$ \\
\hline 23 & $11000-1100 \theta^{*}$ & 51 & $10010-00110$ & 79 & $\theta 110 \theta-\theta 0101$ & 107 & $\theta 0111-\theta 010 \theta$ \\
\hline 24 & $11001-00001$ & 52 & $10010-01001$ & 80 & $\theta 110 \theta-\theta 011 \theta$ & 108 & $\theta 0101-\theta 0011$ \\
\hline 25 & $11001-00010$ & 53 & $10010-01010$ & 81 & $01100-01001$ & 109 & $00101-00101^{*}$ \\
\hline 26 & $11001-00100$ & 54 & $10010-01100$ & 82 & $01100-01010$ & 110 & $00011-00011^{*}$ \\
\hline 27 & $11001-01000$ & 55 & $10010-10001$ & 83 & $\theta 1100-0110 \theta^{*}$ & & \\
\hline 28 & $11001-10000$ & 56 & $10010-10010^{*}$ & 84 & $01101-00001$ & & \\
\hline
\end{tabular}




\subsection{Inadmissible Actuation Scheme}

An inadmissible actuation scheme signifies a choice of actuated joints that render the object uncontrollable. This occurs when for any configuration of the robot arms the global wrench system Eqn. (30) degenerates, i.e. $\operatorname{rank}(\mathrm{W})<6, \operatorname{rank}\left(\mathrm{W}^{c}\right)=2, \operatorname{rank}\left(\mathrm{W}^{a}\right) \leq 4$. In order to search for these schemes using screw theory, descriptions of the actuation and constraint wrenches that hold in any configuration are examined using Eqn. (26), Eqn. (27) and Eqn. (28). For the 71 inadmissible schemes, the reason for the inadmissibility can be divided into two cases.

1. The unique case for actuation scheme number 23 denoted as 11000-11000, where the closed chain can rotate freely about axis $\left(B_{1} B_{2}\right)$.

2. The general case, occurring in 70 of the 210 actuation schemes, where the closed chain can rotate freely about $\operatorname{axis}\left(A_{1} A_{2}\right)$.

Case 1: Scheme no. $2311000-11000$ i.e. $\mathbf{q}_{a}=\left[\begin{array}{llll}q_{1} & q_{2} & q_{6} & q_{7}\end{array}\right]$.

Since it is generally preferable to actuate joints close to the base, the case where the base U-joints are actuated is examined. The global wrench system as illustrated in Fig. 5, is spanned by six pure forces, namely, $\mathrm{W}=\operatorname{span}\left(\zeta_{0 r}^{c} \zeta_{0 l}^{c} \zeta_{01}^{a} \zeta_{02}^{a} \zeta_{06}^{a} \zeta_{07}^{a}\right)$. In this case the six pure forces, $\zeta_{0 r}^{c} \zeta_{0 l}^{c} \zeta_{01}^{a} \zeta_{02}^{a} \zeta_{06}^{a} \zeta_{07}^{a}$, all intersect line $\left(B_{1} B_{2}\right)$. This implies that regardless of the configuration, there exists a zero pitch twist, whose axis is the axis $\left(B_{1} B_{2}\right)$, that is reciprocal to all forces in the global wrench system.

Case 2: Neither joint 1 nor joint 6 is actuated, e.g. Scheme no. 83 0110001100 i.e. $\mathbf{q}_{a}=\left[\begin{array}{llll}q_{2} & q_{3} & q_{7} & q_{8}\end{array}\right]$. The global wrench system as illustrated in Fig. 6, is given by $\mathrm{W}=\operatorname{span}\left(\zeta_{0 l}^{c} \zeta_{0 r}^{c} \zeta_{02}^{a} \zeta_{03}^{a} \zeta_{07}^{a} \zeta_{08}^{a}\right)$. In this case the six pure forces, $\zeta_{0 l}^{c} \zeta_{0 r}^{c} \zeta_{02}^{a} \zeta_{03}^{a} \zeta_{07}^{a} \zeta_{08}^{a}$, all intersect line $\left(A_{1} A_{2}\right)$. This implies that regardless of the configuration, there exists a zero pitch twist, whose axis is the line $\left(A_{1} A_{2}\right)$ and passing through point $A_{1}$, that is reciprocal to all forces of the global wrench system.

\section{Local Motion Analysis Based on Screw Theory}

In this section the local motion of mechanism is obtained by examining the relationship between the constraint forces in different configurations.

When the constraint forces are parallel, i.e., $\zeta_{0 r}^{c} \| \zeta_{0 l}^{c 2}$, there are two independent $\infty$-pitch twists, $\epsilon_{\infty 1}$ and $\epsilon_{\infty 2}$, reciprocal (normal) to $\zeta_{0 r}^{c}$ and $\zeta_{0 l}^{c}$. There are also two independent zeropitch twists, $\epsilon_{01}$ and $\epsilon_{02}$, reciprocal (coplanar) to $\zeta_{0 r}^{c}$ and $\zeta_{0 l}^{c}$ as shown in Fig. 7. Therefore,

\footnotetext{
${ }^{2} \|$ implies the axes of the two screws are parallel, whereas $\Varangle$ implies they are not parallel
} 


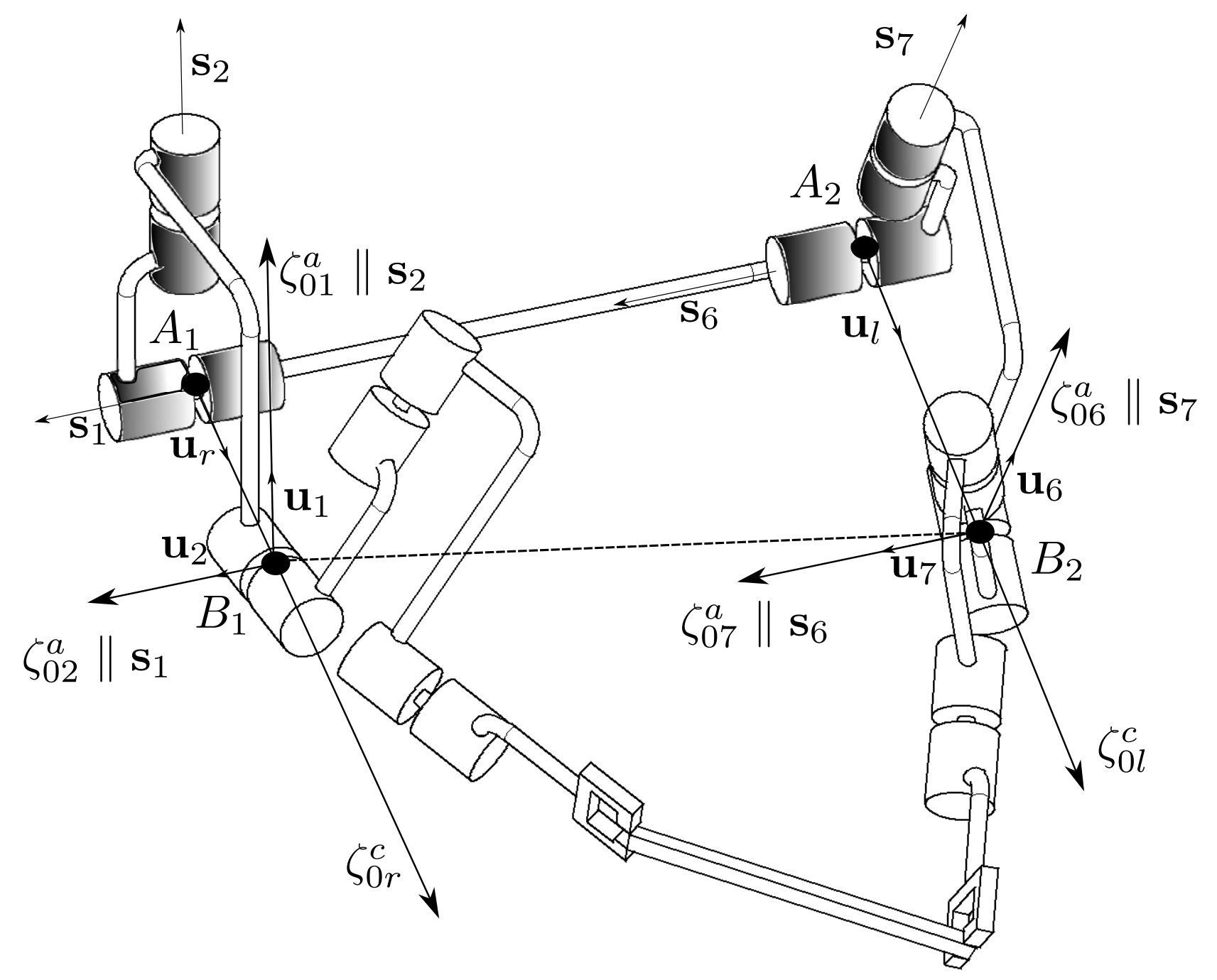

Figure 5: Non-admissible actuation scheme, scheme no. 23

locally the motion can be decomposed into 2 translations normal to the constraint forces and any two linearly independent rotations in the plane formed by the constraint forces. Then the infinitesimal motion type is $2 \mathrm{~T} 2 \mathrm{R}^{3}$.

When $\zeta_{0 r}^{c} \nVdash \zeta_{0 l}^{c}$ but the axes intersect, there is one $\infty$-pitch twist $\epsilon_{\infty 1}$ reciprocal (normal) to both $\zeta_{0 r}^{c}$ and $\zeta_{0 l}^{c}$ and there are three independent zero-pitch twists, $\epsilon_{01}, \epsilon_{02}$ and $\epsilon_{03}$, reciprocal (coplanar) to both $\zeta_{0 r}^{c}$ and $\zeta_{0 l}^{c}$ as shown in Fig. 8. In this case, the object can perform three infinitesimal rotations about the intersection point of the constraint forces and one infinitesimal translation along the normal to both constraint forces. Therefore, the infinitesimal motion type is $1 \mathrm{~T} 3 \mathrm{R}$.

\footnotetext{
${ }^{3} \mathrm{~T}$ and $\mathrm{R}$ stand for Translation and Rotation, respectively.
} 


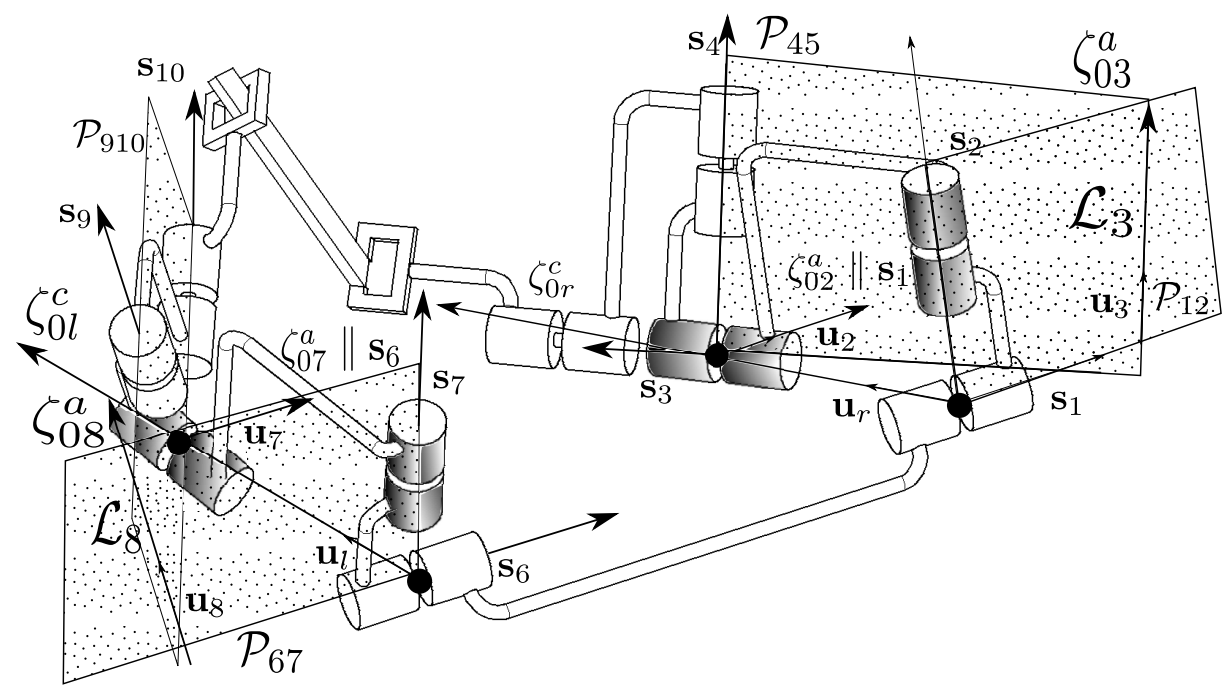

Figure 6: Non-admissible actuation scheme, case 2, scheme no. 83

A final more general case is where the constraint forces are neither parallel nor intersecting as shown in Fig. 9. In this case the infinitesimal motion type is still 1T3R however the three rotational axes do not intersect. It is noteworthy that the set of all lines intersecting two given skew lines generates a linear line variety of dimension 4 called a hyperbolic congruence [9]. As a consequence the object can perform three infinitesimal rotations about three axes that do not intersect and one infinitesimal translation along the direction normal to the two constraint forces.

\section{Singularity Analysis}

This section deals with the singularity analysis of the NAO T14 cooperating arms when it firmly grasps an object. Arm singularities can be characterized by a loss of DOF of the arm, while a gain of DOF or a lack of stiffness of the manipulator occurs in a parallel singular configuration $[9,18]$. 


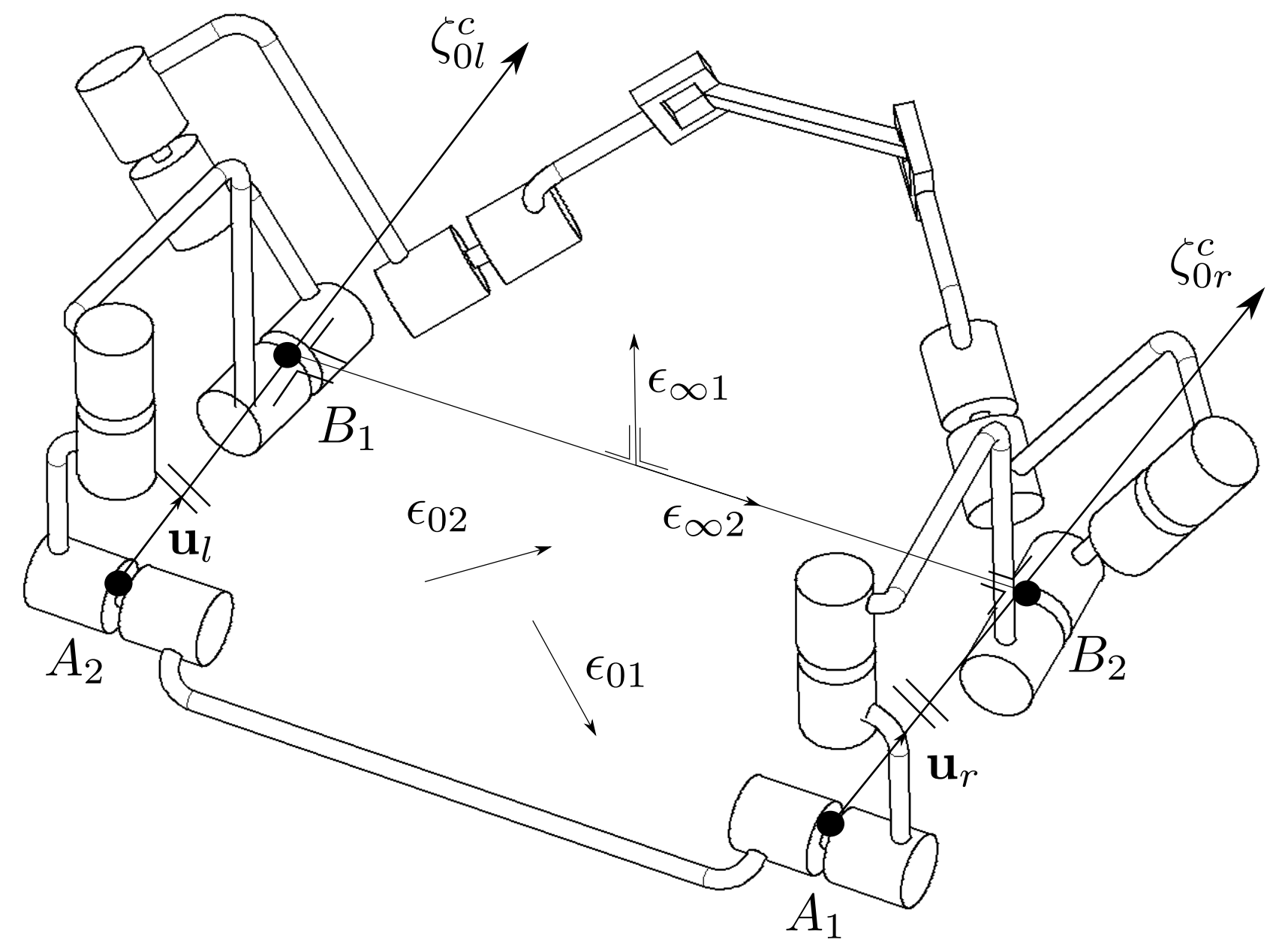

Figure 7: Reciprocal twists to parallel constraint forces (2T2R infinitesimal motion type) 


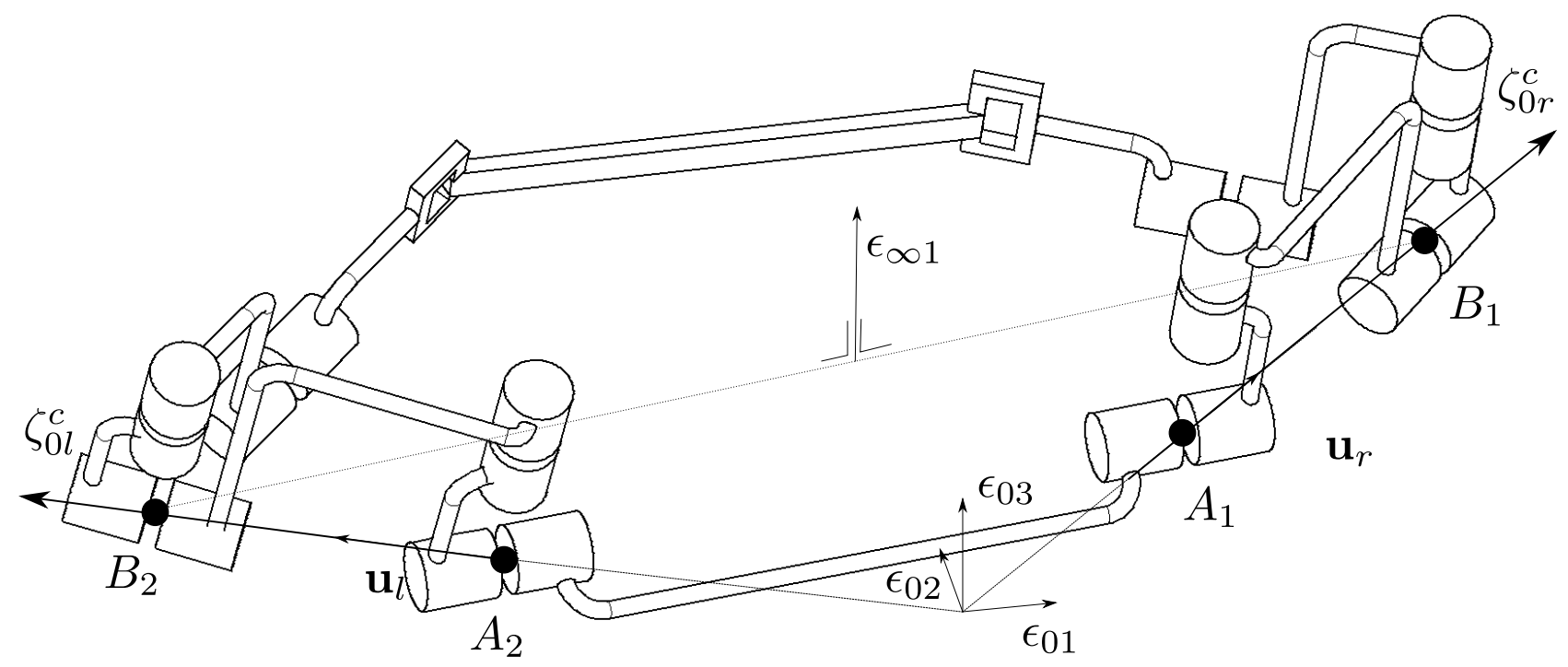

Figure 8: Reciprocal twists to intersecting constraint forces (1T3R infinitesimal motion mode)

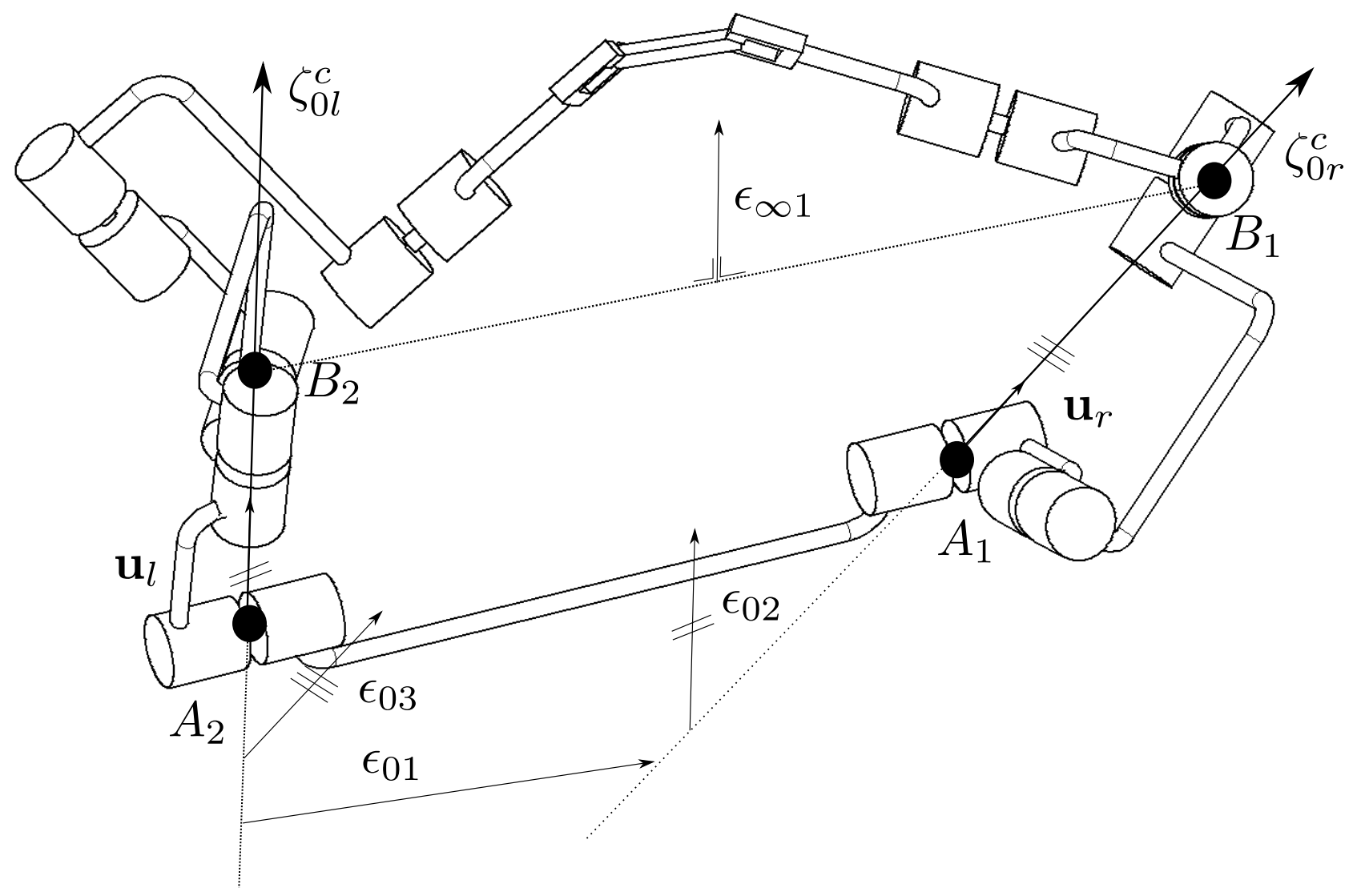

Figure 9: Reciprocal twists to non-intersecting and non parallel constraint forces (1T3R infinitesimal motion mode) 


\subsection{Arm Singularities}

An arm singularity is similar to the singularity of a serial manipulator. It occurs for the dualarm system when the arm kinematic screw system (twist system) degenerates. Consequently, the grasped object loses one or more DOF in such a configuration. From Eqn. (20) the kinematic Jacobian matrix of the right arm can be written as:

$$
\mathbf{J}_{r}=\left[\begin{array}{ccccc}
\mathbf{s}_{1} & \mathbf{s}_{2} & \mathbf{s}_{3} & \mathbf{s}_{4} & \mathbf{s}_{5} \\
\mathbf{a}_{1} \times \mathbf{s}_{1} & \mathbf{a}_{1} \times \mathbf{s}_{2} & \mathbf{b}_{1} \times \mathbf{s}_{3} & \mathbf{b}_{1} \times \mathbf{s}_{4} & \mathbf{b}_{1} \times \mathbf{s}_{5}
\end{array}\right]
$$

To simplify the analysis, the origin of frame $\hat{\mathcal{F}}_{\text {obj }}$ is transformed to the S-joint center. Eqn. (32) becomes:

$$
\mathbf{J}_{r}=\left[\begin{array}{ccccc}
\mathbf{s}_{1} & \mathbf{s}_{2} & \mathbf{s}_{3} & \mathbf{s}_{4} & \mathbf{s}_{5} \\
\mathbf{a}_{1} \times \mathbf{s}_{1} & \mathbf{a}_{1} \times \mathbf{s}_{2} & \mathbf{0}_{3} & \mathbf{0}_{3} & \mathbf{0}_{3}
\end{array}\right]
$$

The right arm reaches a limb singularity when $\mathbf{J}_{r}$ is rank deficient. There are two possible singular configurations leading to rank deficiency. Firstly when $\mathbf{s}_{3}$ is parallel to $\mathbf{s}_{5}$ obtained at $\theta_{4}=0 \pm \pi$. Secondly when the S-joint center lies on the line passing through point $A_{1}$ and parallel to $\mathbf{s}_{1}$, meaning $\mathbf{s}_{1} \times \mathbf{a}_{1}=\mathbf{0}_{3}$. This configuration occurs at $\theta_{2}=\operatorname{atan}\left(\frac{-r_{3}}{d_{3}}\right)$.

\section{The effect of arm singularities in closed-loop}

The serial singularity in closed-loop means that the object loses 1-DOF regardless of the actuation scheme. This is due to the fact that there are now three independent constraint wrenches applied on the object $\zeta_{0 r}^{c}, \zeta_{0 l}^{c}$ and the wrench due to the singularity. However the actuation scheme affects whether or not there is internal motion in the mechanism, i.e., if a link can move locally without affecting the pose of the object. This type of local motion is known as an inner singularity.

In summary, for the serial singularity condition $\theta_{4}=0$, when neither joint 3 nor joint 5 are actuated, the global wrench system degenerates and Link 4 can move freely. Equally in the

second serial singularity case $\theta_{2}=\operatorname{atan}\left(\frac{-r_{3}}{d_{3}}\right)$, the linearly dependent joints are joints $1,3,4$ and 5. Again, if none of these joints are actuated during the singular configuration, an inner singularity will occur.

Example: Scheme no. $1211010-10000$ i.e. $\mathbf{q}_{a}=\left[\begin{array}{llll}q_{1} & q_{2} & q_{4} & q_{6}\end{array}\right]$. When the arm is in a serial configuration $\theta_{4}=0$, we propose to analyze the mechanism by breaking the chain at Link 4, separating the two linearly dependent joints as shown in Fig. 10. By breaking the chain 
in this way, Link 4 becomes analogous to the object and the procedure outlined in Section 4.2 can be reapplied. If the loop is broken so the two linearly independent joints remain on the same serial chain, this procedure breaks down. Hence, in this case the twist system associated with the right and the left chains are:

$$
\begin{aligned}
& \mathrm{T}^{\mathrm{r}}=\operatorname{span}\left(\nu_{01}, \quad \nu_{02}, \quad \nu_{03}, \quad \nu_{04}\right) \\
& \mathrm{T}^{1}=\operatorname{span}\left(\nu_{06}, \quad \nu_{07}, \quad \nu_{08}, \quad \nu_{09}, \quad \nu_{010}, \quad \nu_{05}\right)
\end{aligned}
$$

The left arm is now composed of six joints while the right arm is composed of four joints. Thus there are no constraint forces associated with the $\mathrm{T}^{1}$, meanwhile there are now two constraint wrenches associated with the right arm: $\zeta_{0 r 1}^{c}$ the constraint wrench expressed in Eqn.(24), and $\zeta_{0 r 2}^{c}$ is equivalent to $\zeta_{05}^{a}$ from Eqn.(27b) since it should be reciprocal to $\nu_{01}, \nu_{02}, \quad \nu_{03}, \quad \nu_{04}$, hence

$$
\zeta_{0 r 1}^{c}=\left[\begin{array}{c}
\mathbf{u}_{r} \\
\mathbf{b}_{\mathbf{1}} \times \mathbf{u}_{r}
\end{array}\right] \quad \text { (36a) } \quad \zeta_{0 r 2}^{c}=\left[\begin{array}{c}
\mathbf{u}_{5} \\
\mathbf{a}_{\mathbf{1}} \times \mathbf{u}_{5}
\end{array}\right]
$$

The first, second and fourth joints are actuated, therefore the actuation wrench system is spanned by the following pure forces:

$$
\begin{aligned}
& \zeta_{01}^{a}=\left[\begin{array}{c}
\mathbf{u}_{1} \\
\mathbf{b}_{\mathbf{1}} \times \mathbf{u}_{1}
\end{array}\right] \\
& \zeta_{04}^{a}=\left[\begin{array}{c}
\mathbf{u}_{4 s} \\
\mathbf{b}_{\mathbf{1}} \times \mathbf{u}_{4 s}
\end{array}\right] \\
& \zeta_{02}^{a}=\left[\begin{array}{c}
\mathbf{u}_{2} \\
\mathbf{b}_{\mathbf{2}} \times \mathbf{u}_{2}
\end{array}\right]
\end{aligned}
$$

$\mathbf{u}_{4 s}$ is the unit vector of the line passing through point $A_{1}$ and parallel to $\mathbf{s}_{3}$. Joint 6 , of the left arm is actuated, therefore the actuation wrench system is spanned by the following finite pitch wrench, namely:

$$
\zeta_{06}^{a}=\left[\begin{array}{c}
\mathbf{u}_{6 s} \\
\mathbf{r}_{6 s} \times \mathbf{u}_{6 s}+\lambda_{6 s} \mathbf{u}_{6 s}
\end{array}\right]
$$

$\mathbf{u}_{6 s}$ is the unit vector of the screw reciprocal to $\nu_{07}, \nu_{08}, \quad \nu_{09}, \quad \nu_{010}, \quad \nu_{05}$, while $\mathbf{r}_{6 s}$ is a vector pointing from any point on this axis to the origin.

As a result, the global wrench system applied on $\operatorname{Link} 4$ is spanned by $\zeta_{0 r 1}^{c}, \zeta_{0 r 2}^{c}, \zeta_{01}^{a}, \zeta_{02}^{a} \zeta_{04}^{a}$, 
$\zeta_{\lambda 6}^{a}$, namely:

$$
\mathrm{W}=\operatorname{span}\left(\zeta_{0 r 1}^{c}, \quad \zeta_{0 r 2}^{c}, \quad \zeta_{01}^{a}, \quad \zeta_{02}^{a}, \quad \zeta_{04}^{a}, \quad \zeta_{\lambda 6}^{a}\right)
$$

The singularity condition means that $\nu_{03}$ and $\nu_{05}$ are linearly dependent, therefore logically any screw that is reciprocal $\nu_{03}$ is reciprocal to $\nu_{05}$ and vice versa. It follows that with this choice of actuators, $\nu_{03}$ and $\nu_{05}$ are reciprocal to all the wrenches of the global wrench system.

Using Eqn. (19), Eqn. (36), Eqn. (37), and Eqn. (38):

$$
\left[\begin{array}{cc}
\mathbf{b}_{\mathbf{1}} \times \mathbf{u}_{r} & \mathbf{u}_{r} \\
\mathbf{a}_{\mathbf{1}} \times \mathbf{u}_{5} & \mathbf{u}_{5} \\
\mathbf{b}_{\mathbf{1}} \times \mathbf{u}_{1} & \mathbf{u}_{1} \\
\mathbf{b}_{\mathbf{2}} \times \mathbf{u}_{2} & \mathbf{u}_{2} \\
\mathbf{b}_{\mathbf{1}} \times \mathbf{u}_{4 s} & \mathbf{u}_{4 s} \\
\mathbf{r}_{\mathbf{7}} \times \mathbf{u}_{7 s}+\lambda \mathbf{u}_{7 s} & \mathbf{u}_{7 s}
\end{array}\right]\left[\begin{array}{c}
\mathbf{s}_{3} \\
\mathbf{b}_{\mathbf{1}} \times \mathbf{s}_{3}
\end{array}\right]=0
$$

Hence the null space is spanned by $\nu_{03}$ and $\nu_{05}$. Since the null space exists, the global wrench system from Eqn. (39) must be rank deficient, and unable to fully constrain Link 4.

\subsection{Parallel Singularities}

\section{Constraint Singularities}

A constraint singularity occurs when the constraint wrench system (26) degenerates, i.e., when $\zeta_{0 r}^{c}$ and $\zeta_{0 l}^{c}$ are linearly dependent. The closed-loop system reaches such a configuration when the two S-joint centers lie on $\mathbf{s}_{1}$ and $\mathbf{s}_{6}$, namely:

$$
\theta_{2}=\operatorname{atan}\left(\frac{-r_{3}}{d_{3}}\right) \text { and } \theta_{7}=\operatorname{atan}\left(\frac{r_{3}}{d_{3}}\right)
$$

It is noted that when the closed-loop system reaches a constraint singularity, both arms are in arm singularity configuration as described in Section 6. From Section 6.1, it can be seen that an arm singularity in a serial mechanism increases the degree of the constraint wrench system. Let the constraint wrench due to the serial singularities of the right and left arm be denoted as $\zeta_{0 s 1}$ and $\zeta_{0 s 2}$ respectively, which are obtained as:

$$
\zeta_{0 s r}^{c}=\left[\begin{array}{c}
\mathbf{u}_{\mathbf{1}} \\
\mathbf{b}_{\mathbf{1}} \times \mathbf{u}_{\mathbf{1}}
\end{array}\right]
$$




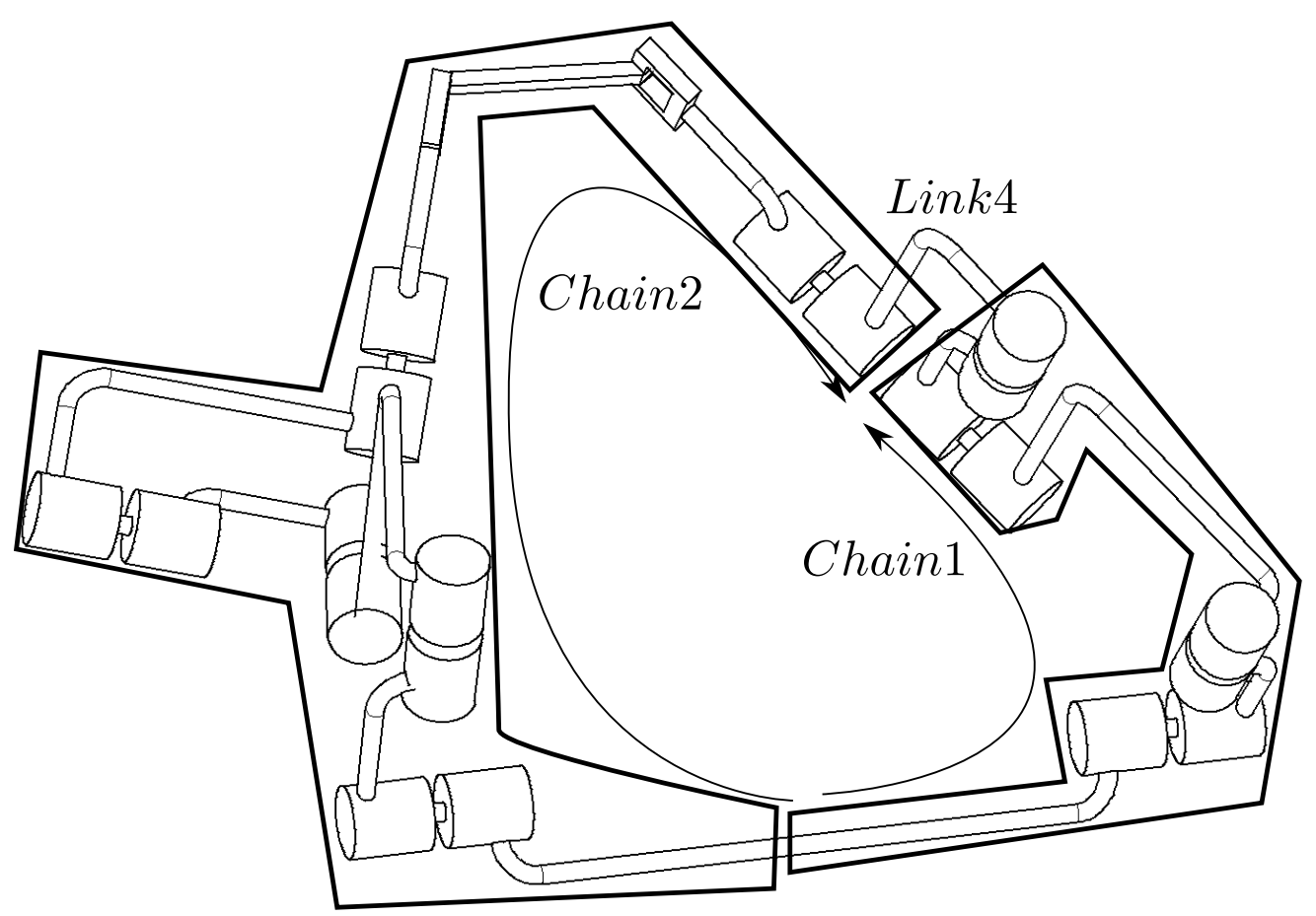

Figure 10: Serial chains for inner singualrity analysis 


$$
\zeta_{0 s l}^{c}=\left[\begin{array}{c}
\mathbf{u}_{6} \\
\mathbf{b}_{2} \times \mathbf{u}_{6}
\end{array}\right]
$$

Hence at the studied configuration, four wrenches forming a 3-system as described in Fig. 11 are applied on the object: the constraint wrenches $\zeta_{0 r}^{c}$ and $\zeta_{0 l}^{c}$ and the wrenches due to the serial singularity of each arm $\zeta_{0 s r}^{c}$ and $\zeta_{0 s l}^{c}$. Consequently, the object has 3-DOF in this configuration.

The infinitesimal motion type varies depending on the relationship between $\zeta_{0 s r}^{c}$ and $\zeta_{0 s l}^{c}$. If they are parallel, there is one $\infty$-pitch twists, $\epsilon_{\infty}$ reciprocal (normal) to $\zeta_{0 r}^{c}, \zeta_{0 l}^{c}, \zeta_{0 s r}^{c}$ and $\zeta_{0 s l}^{c}$ while there are two independent zero-pitch twists, $\epsilon_{01}$ and $\epsilon_{02}$, reciprocal (coplanar) to $\zeta_{0 r}^{c}$, $\zeta_{0 l}^{c}$, $\zeta_{0 s r}^{c}$ and $\zeta_{0 s l}^{c}$. Therefore, locally the motion can be decomposed into 1 translation normal to the constraint forces and any two linearly independent rotations in the plane formed by the constraint forces. Then the infinitesimal motion type is 1T2R.

Contrarily if $\zeta_{0 s r}^{c}$ and $\zeta_{0 s l}^{c}$ are not parallel, there are three independent zero-pitch twists, $\epsilon_{01}$, $\epsilon_{02}$ and $\epsilon_{03}$ reciprocal (coplanar) to $\zeta_{0 r}^{c}, \zeta_{0 l}^{c}, \zeta_{0 s r}^{c}$ and $\zeta_{0 s l}^{c}$. In this configuration the infinitesimal motion type is $0 \mathrm{~T} 3 \mathrm{R}$.

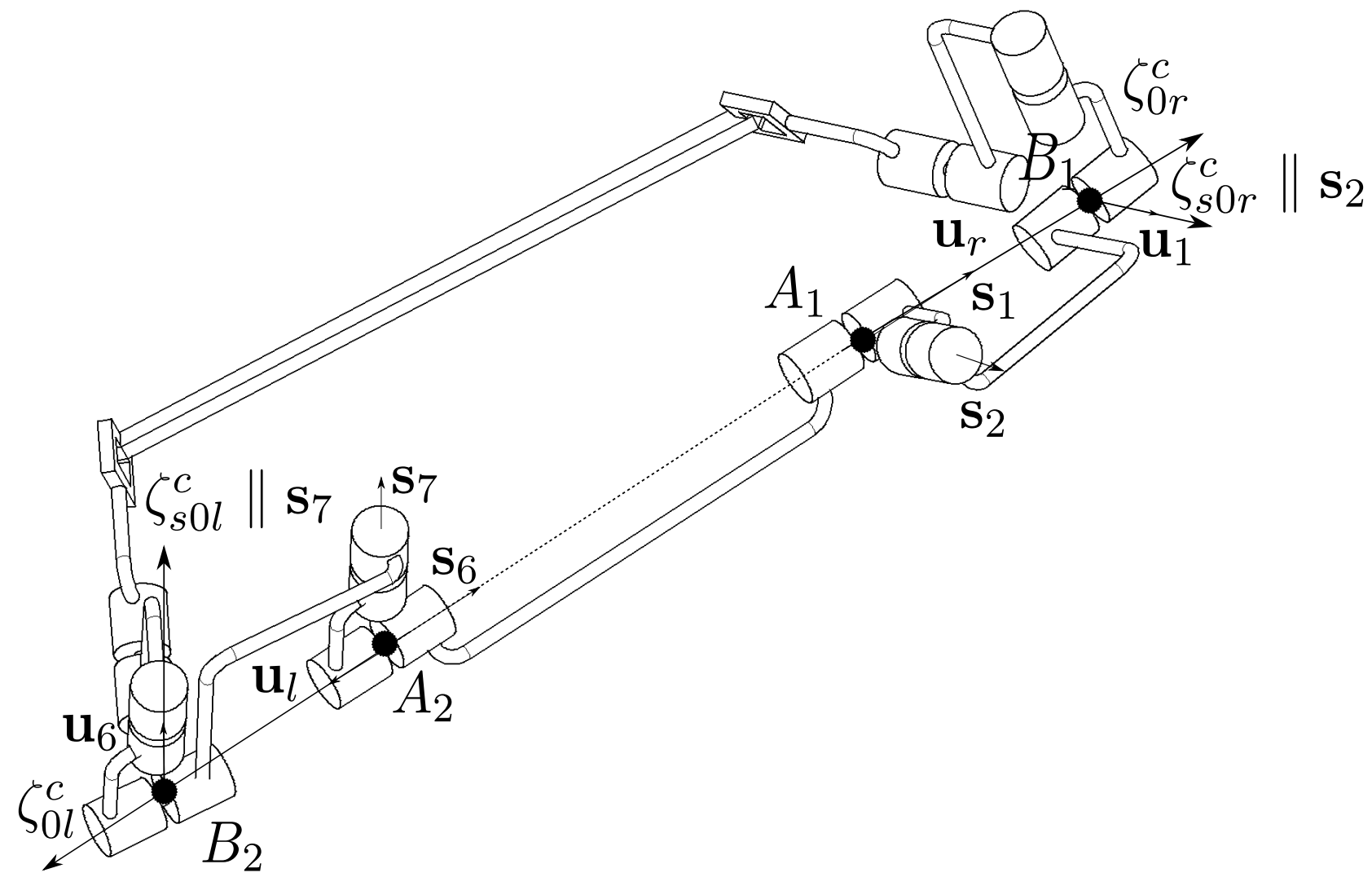

Figure 11: Constraint singularity of the dual-arm system 


\section{Actuation Singularities:}

The choice of actuators means that the object can be controlled but may encounter actuation singularities at certain configurations. An actuation singularity occurs when the global wrench system (30) degenerates while the constraint wrench system (26) does not. Due to the large number of viable actuation schemes, each presumably containing several actuation singularities, a more general illustration is presented below.

Example Actuation Singularity 1: Considering the configuration $\theta_{1}=\theta_{6}$ for any actuation scheme where $\left[\begin{array}{ll}q_{2} & q_{7}\end{array}\right] \in \mathbf{q}_{a}$

The two actuation wrenches associated with joints 2 and $7, \zeta_{02}^{a}$ and $\zeta_{07}^{a}$, and the two constraint wrenches associated with the systems geometry $\zeta_{0 l}^{c}$ and $\zeta_{0 r}^{c}$, normally constitute a 4system. When $\theta_{1}=\theta_{6}$, the unit vectors $\mathbf{u}_{\mathbf{r}}, \mathbf{u}_{\mathbf{l}}, \mathbf{u}_{\mathbf{2}}, \mathbf{u}_{\mathbf{7}}$ all lie in $\mathcal{P}$, where $\mathcal{P}$ is the plane containing points $A_{1}, B_{1}, A_{2}, B_{2}$. The global wrench system degenerates due to the linear dependence of the wrenches $\zeta_{02}^{a}, \zeta_{07}^{a}, \zeta_{0 l}^{c}$ and $\zeta_{0 r}^{c}$. In this case there are three twists reciprocal to all the wrenches. For any choice of the remaining two actuators, the global wrench system will degenerate. One such example of this degeneracy is found in scheme $5, \mathbf{q}_{a}=\left[q_{1}, q_{2}, q_{3}, q_{7}\right]$ 11100 - 01000. The actuation singularity is shown in Fig. 12.

Example Actuation Singularity 2 Considering the configuration $\theta_{1}=\theta_{6}$ and $\theta_{3}=\pi / 2$ for any actuation scheme where either joint 2 or 7 , and joint 5 are actuated.

In this configuration the actuation wrench associated with joint 5 lies in the plane $\mathcal{P}$, where $\mathcal{P}$ is the plane containing points $A_{1}, B_{1}, A_{2}, B_{2}$. The global wrench system degenerates due to the linear dependence of the wrenches $\zeta_{02}^{a}\left(\right.$ or $\left.\zeta_{07}^{a}\right), \zeta_{05}^{a}, \zeta_{0 l}^{c}$ and $\zeta_{0 r}^{c}$. One such example of this degeneracy is found in scheme $7, \mathbf{q}_{a}=\left[q_{1}, q_{2}, q_{3}, q_{5}\right] 11101-00000$. The actuation singularity is shown in Fig. 13.

\section{Comparison of Screw theory and Jacobian Methods}

In this section a comparison is made between existing numerical methods, derived in Section 2, classically used to kinematically analyze the singularities of the system and the screw theory methods demonstrated in the previous sections.

Table 3 analyzes the singular configurations obtained in Section 6, with respect to the constraint equations. In [19] singular conditions are defined with respect to the Jacobian matrices. A degeneration of $\mathbf{J}_{\text {act }}$ from (15) is an unmanipulable singularity. By obtaining the null space of $\mathbf{G}_{p}$, denoted as $N\left(\mathbf{G}_{p}\right)$, parallel singularities can be investigated. $\mathbf{J}_{p} \cdot N\left(\mathbf{G}_{p}\right) \neq 0$ 


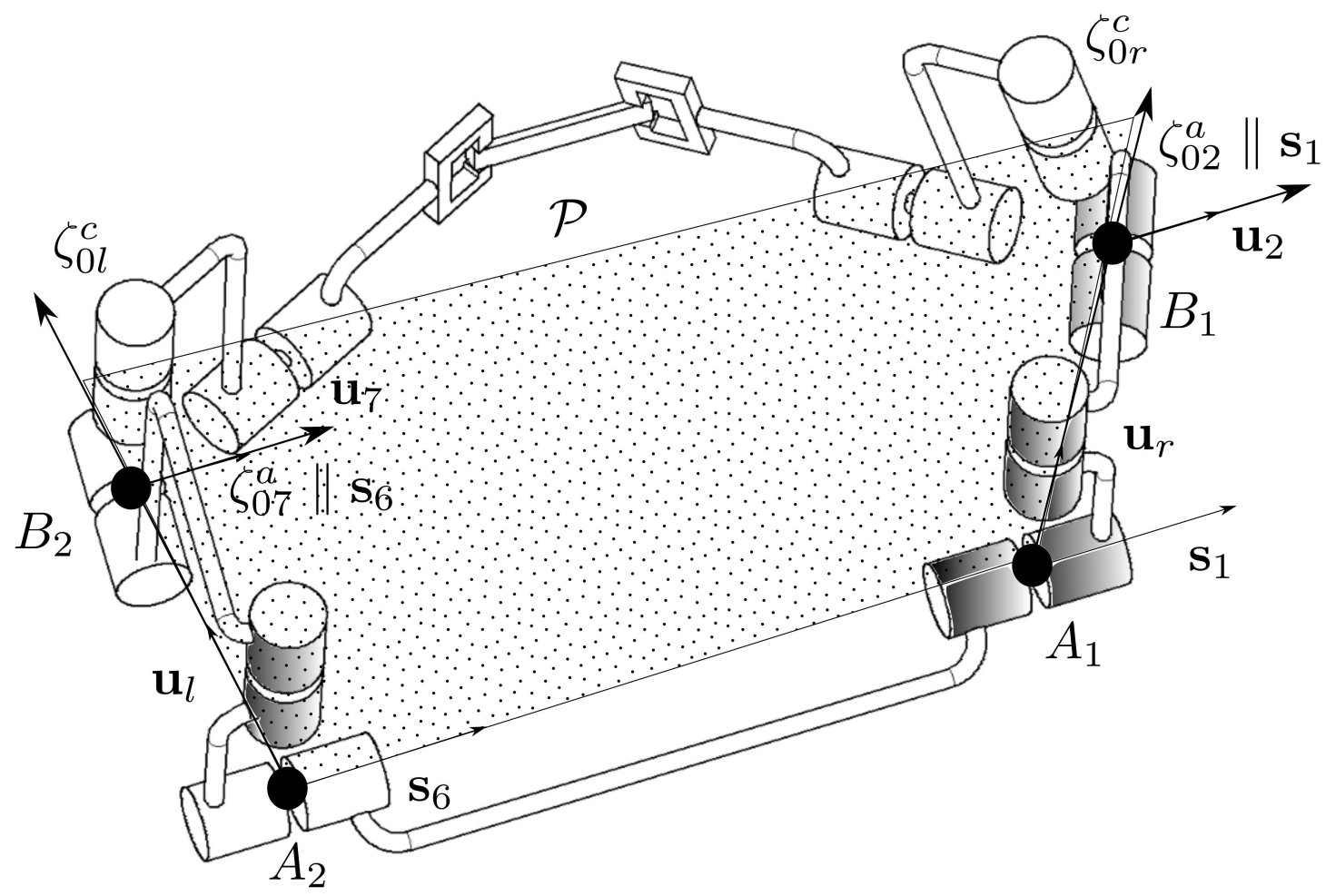

Figure 12: Actuation singularity for scheme no. 5

corresponds to an unstable singularity (a loss of stiffness in the platform). $\mathbf{J}_{p} \cdot N\left(\mathbf{G}_{p}\right)=0$ corresponds to a self motion or inner singularity. To find the inadmissible actuation schemes the matrix $\mathbf{G}_{p}$ is examined. If this matrix is rank deficient for every value of $\mathbf{q}_{a}, \mathbf{q}_{p}$, and $\mathbf{q}_{c}$ the actuation scheme is inadmissible.

Table 3 validates the screw theory analysis on the system notably with respect to serial singularities. For example it shows that by changing the actuation scheme, the lack of stiffness in the system when $\mathbf{J}_{p} \cdot N\left(\mathbf{G}_{p}\right)=0$ can be avoided.

\section{Dynamic Performance of Actuation Schemes}

In this section, firstly the dynamic model of closed loop robots is given. Then the dynamic performance of admissible actuation schemes is compared when the closed chain system is transporting an object in space. 


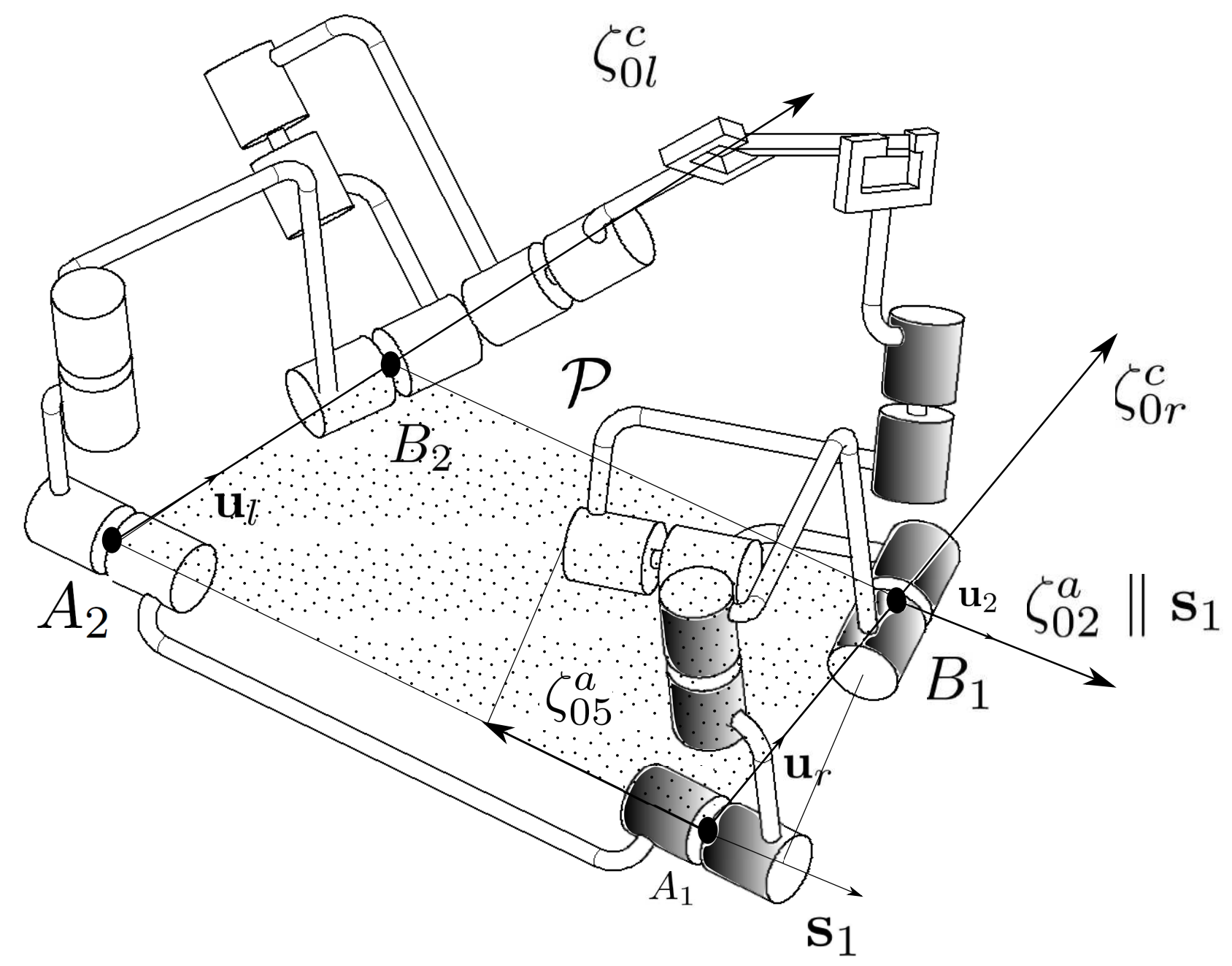

Figure 13: Actuation singularity for scheme no. 7

\subsection{Dynamic Modeling of Closed Loop}

The inverse dynamic model (IDM) calculates the active motor torques $\tau$ in terms of $\mathbf{q}$, $\dot{\mathbf{q}}$ and $\ddot{\mathbf{q}}$ of the joints. Let $\mathbf{q}_{t r}=\left[\begin{array}{ll}\mathbf{q}_{a}^{T} & \mathbf{q}_{p}^{T}\end{array}\right]^{T}$ denote the joint variables of the tree structure. In order to find the Closed Loop Inverse Dynamic Model (CLIDM), first the IDM for the tree structure is found and then converted to CLIDM by using the following relation [20]:

$$
\tau=\left(\frac{\partial \mathbf{q}_{t r}}{\partial \mathbf{q}_{a}}\right)^{T} \Gamma_{t r}=\Gamma_{a}+\left(\frac{\partial \mathbf{q}_{p}}{\partial \mathbf{q}_{a}}\right)^{T} \Gamma_{p}
$$


Table 3: Numerical Status at Singularity

\begin{tabular}{|c|c|c|c|c|c|}
\hline Conditions & No. & Actuation Scheme & $\operatorname{rank}\left(\mathbf{J}_{a c t}\right)$ & $\operatorname{rank}\left(\mathbf{G}_{p}\right)$ & $\overline{\mathbf{J}_{p} \cdot N\left(\mathbf{G}_{p}\right)}$ \\
\hline Benchmark & 4 & $11100-00100$ & 4 & 5 & \{\} \\
\hline Inadmissible & & & & & \\
\hline Scheme 1 & 23 & $11000-11000$ & 4 & 4 & $\neq 0$ \\
\hline Inadmissible & & & & & \\
\hline Scheme 2 & 83 & $01100-01100$ & 4 & 4 & $\neq 0$ \\
\hline Serial & & & & & \\
\hline Singularity $1 \theta_{4}=0$ & 12 & $11010-10000$ & 4 & 4 & $=0$ \\
\hline Serial & & & & & \\
\hline Singularity $1 \theta_{4}=0$ & 13 & $11011-00000$ & 3 & 5 & \{\} \\
\hline Serial & & & & & \\
\hline Singularity $2 \theta_{7}=\operatorname{atan}\left(r_{3} / d_{3}\right)$ & 11 & $11010-01000$ & 4 & 4 & $=0$ \\
\hline Serial & & & & & \\
\hline Singularity $2 \theta_{7}=\operatorname{atan}\left(r_{3} / d_{3}\right)$ & 12 & $11010-10000$ & 3 & 5 & \{\} \\
\hline Actuation & & & & & \\
\hline Singularity $1 \theta_{1}=\theta_{6}$ & 5 & $11100-01000$ & 4 & 4 & $\neq 0$ \\
\hline Actuation & & & & & \\
\hline Singularity $2 \theta_{1}=\theta_{6}, \theta_{3}= \pm \frac{\pi}{2}$ & 7 & $11101-00000$ & 4 & 5 & $\neq 0$ \\
\hline Constraint $\quad \theta_{2}=\operatorname{atan}\left(r_{3} / d_{3}\right)$, & & & & & \\
\hline Singularity $\quad \theta_{7}=\operatorname{atan}\left(r_{3} / d_{3}\right)$ & 5 & $11100-01000$ & 3 & 4 & $=0$ \\
\hline $\begin{array}{ll}\text { Constraint } & \theta_{2}=\operatorname{atan}\left(-r_{3} / d_{3}\right), \\
\text { Singularity } & \theta_{7}=\operatorname{atan}\left(r_{3} / d_{3}\right)\end{array}$ & 4 & $11100-00100$ & 3 & 4 & $\neq 0$ \\
\hline
\end{tabular}

where $\Gamma_{t r}$ denotes the joint torques of the tree structure. It can be expressed as:

$$
\Gamma_{t r}=\left[\begin{array}{c}
\Gamma_{a} \\
\Gamma_{p}
\end{array}\right]=\mathbf{A}_{t r}\left(\mathbf{q}_{t r}\right)\left[\begin{array}{c}
\ddot{\mathbf{q}}_{a} \\
\ddot{\mathbf{q}}_{p}
\end{array}\right]+\mathbf{H}_{t r}\left(\mathbf{q}_{t r}, \dot{\mathbf{q}}_{t r}\right)
$$

$\Gamma_{a}$ and $\Gamma_{p}$ represent the torque on the actuated and passive joints of the tree structure, respectively. $\mathbf{A}_{t r}$ and $\mathbf{H}_{t r}$ are tree structure inertia matrix and the tree structure matrix of Coriolis, Centrifugal and Gravity forces respectively. The inertial parameters of the closed chain system are given in the appendix. Using (10) and (44), we obtain:

$$
\tau=\left[\begin{array}{lll}
\mathbf{I}_{N} & \left(-\mathbf{G}_{p}^{-1} \mathbf{G}_{a}\right)^{T}
\end{array}\right]\left[\begin{array}{c}
\Gamma_{a} \\
\Gamma_{p}
\end{array}\right]
$$

$\mathbf{I}_{N}$ is the identity matrix of dimension $N$, where $N$ is equal to the DOF of the system. Substituting the general expression for the tree dynamic model given by (45) into (44), the closed 
loop dynamic model is obtained as:

$$
\tau=\left[\begin{array}{ll}
\mathbf{I}_{N} & \left(-\mathbf{G}_{p}^{-1} \mathbf{G}_{a}\right)^{T}
\end{array}\right] \mathbf{A}_{t r}\left[\begin{array}{c}
\ddot{\mathbf{q}}_{a} \\
\ddot{\mathbf{q}}_{p}
\end{array}\right]+\left[\begin{array}{ll}
\mathbf{I}_{N} & \left(-\mathbf{G}_{p}^{-1} \mathbf{G}_{a}\right)^{T}
\end{array}\right] \mathbf{H}_{t r}
$$

The direct dynamic model (DDM) calculates the independent joint accelerations $\ddot{\mathbf{q}}_{a}$ from the motor torques $\tau$. It can be obtained after substituting $\ddot{\mathbf{q}}_{p}$ in terms of $\ddot{\mathbf{q}}_{a}$ using (9) in (47) and solving the obtained expression to obtain $\ddot{q}_{a}$.

\subsection{Dynamic Performance}

The comparison criterion is taken as the integral of the sum of squares of the motorised torques, which indicates the motor's power loss [21]. The power loss of a motor is proportional to the square of the current flowing through it. Since the torque of the motor is directly proportional to the current, a power loss criterion given in (48), is defined from time $=0$ to time $=t$, where $\eta$ has units $N^{2} m^{2} s$. The motor torques can be calculated using (47).

To facilitate the presentation, it is supposed that joint 10 is always modeled as the cut joint, therefore actuation schemes containing joint 10 are excluded in this configuration. ${ }^{4}$ However in order to maximize the number of schemes that are tested, the symmetric equivalent of the these schemes are included. To illustrate this point, take scheme no. 2, 11100-00001 . By cutting the chain at joint 10 this actuation scheme cannot be realized, instead the scheme 00001-11100 is used. The two schemes will have the same performance when tested over many random trajectories. In total 64 actuation schemes are tested, (there are 7 kinematically admissible schemes that remain untested).

$$
\eta=\int_{0}^{t} \tau^{T} \tau d t
$$

Figure 14 shows the results for three hundred trajectories. The $x$-axis gives the scheme number and the $y$-axis measures the number of trajectories. For each simulation an arbitrarily shaped rigid object is grasped by the two arms, the object is transported along a spatial trajectory by the cooperative system in a fixed time $t_{\text {final }}$. The trajectory is defined between two points in the joint space using a fifth degree polynomial. It is continuous in both velocity and acceleration. The simulation is repeated using the same object and the same trajectory, for each actuation scheme (64 times). Three hundred simulations are executed. Each simulation

\footnotetext{
${ }^{4}$ To actuate $q_{10}$ a new tree structure robot must be defined.
} 


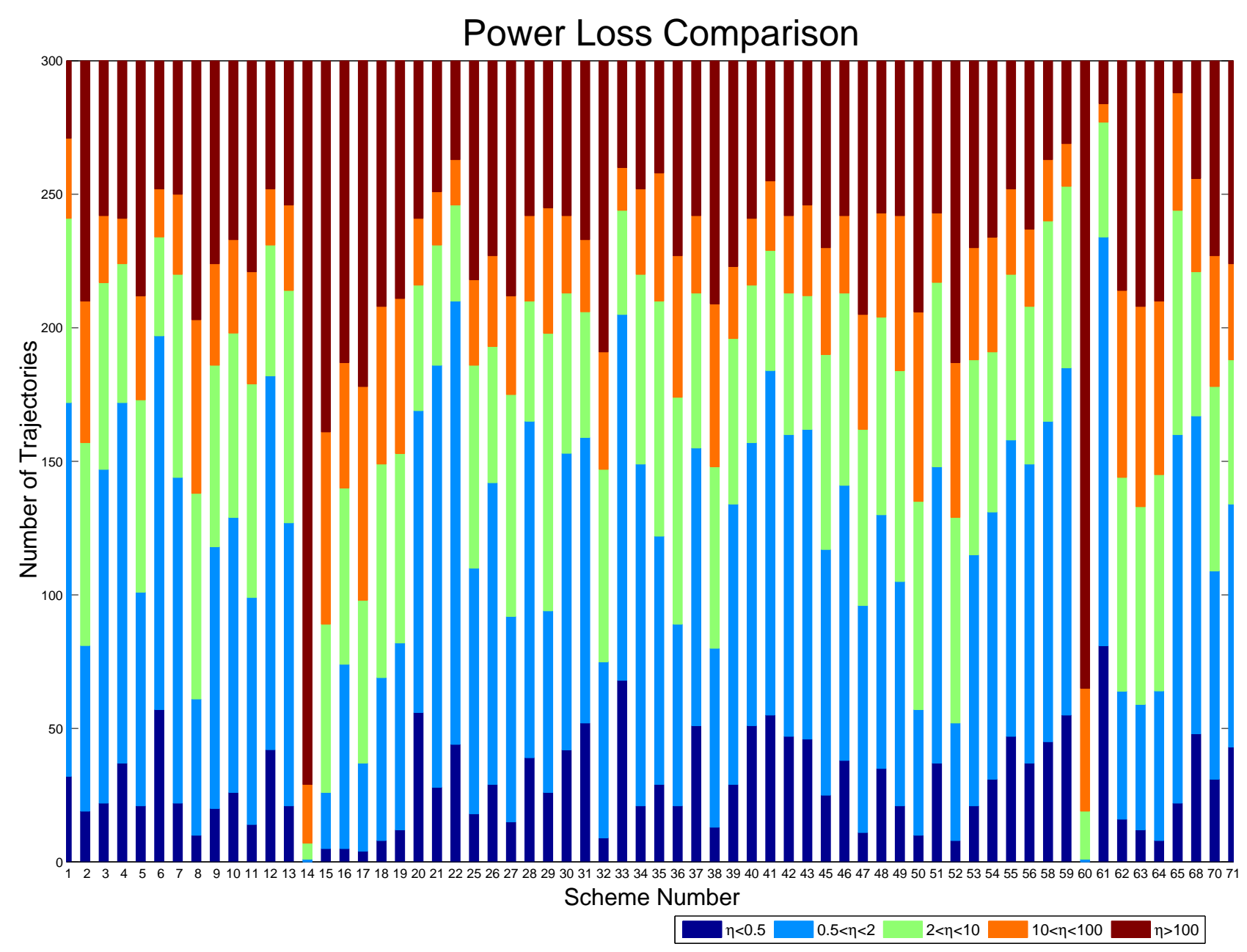

Figure 14: Power Loss for Random trajectories

uses a new object and a new spatial path. A mathematical description of the trajectory is given in appendix.

The chart shows how often $\eta$ lies between the defined bands. For example, taking actuation scheme 1 11110 - 00000, from the three hundred trajectories, $\eta<0.5$ in 32 cases, $0.5<\eta<2$ in 140 cases, $2<\eta<10$ in 69 cases, $10<\eta<100$ in 30 cases and $\eta>100$ in 29 cases. Obviously the most attractive cases are those where $\eta<0.5$.

The chart clearly shows a disparity of $\eta$ between the schemes, notably no 60, 10011 01000 and no 61, 10011-10000. In this case, the change of one actuator has a large effect on the resulting dynamic performance, increasing the number of trajectories where the $\eta>100$ from 16 to 235. Furthermore a large decrease is seen in the trajectories that have a very good performance from 81 to 0. A similar phenomenon can be seen for schemes no 11, 11010- 
- 01000 and no 12, 11010 - 10000. Generally it can be seen that best performing schemes contain actuated joints 1 and 6 , the base joints.

In Fig.15, the number of trajectories where the required actuated joint torque is greater than the motor's peak torque (as given in the appendix) are shown. For example, taking actuation scheme 1 11110 - 00000, from the three hundred trajectories, 43 require a torque greater than the peak torque.

In terms of best performing schemes, this chart correlates with the results seen in Fig.14. In contrast, however this chart, shows the effect of the lower torque limit for joint 5 . For instance, from Fig.14, it is seen that scheme no. 71 has an acceptable dynamic performance. However Fig.15 shows a high number of violations of nominal torque.

The torque required is proportional to the desired acceleration, therefore by increasing $t_{\text {final }}$, the time taken to complete the trajectory, the maximum torque can be reduced. Taking this into account, Fig.15 also indicates the actuation scheme that are capable of transporting the object in the least amount of time without violating the motor constraints.

\section{Actuation Scheme Selection}

The use of any inadmissible scheme can be immediately ruled out. As we have seen in Section 4.2, there are 39 such schemes in which the object has an uncontrollable DOF. Recall that to avoid this scenario, either joint 1 or joint 6 must be actuated. The joint configuration, $\theta_{1}=\theta_{6}$ generates an actuation singualrity when both $q_{2}$ and $q_{7}$ are actuated. This would restrict the range of the base joints leading to a large reduction in the workspace. By actuating either $q_{3}$ or $q_{5}$ on the right arm and $q_{8}$ or $q_{10}$ on the left arm, the lack of stiffness linked to the inner singularity can be avoided. This lack of stiffness will occur when either of the arms pass through the arm singularity.

From studying the results of the power loss test, it is clear that the best performing schemes actuate both joint 1 and joint 6. Moreover there is a clear and logical tendency for schemes that contain actuators near the base to preform better, for example scheme number 6, 11010-10000 and scheme number 22, 11000-10100. Finally, Fig. 15, clearly shows that if the real motor parameters are taken into account, schemes that actuate either joint 5 or joint 10 should be avoided.

In order to propose an optimum actuation scheme, both the kinematic and dynamic considerations must be used. As shown in Fig.14, actuation scheme 41, 10100-10001 has a good dynamic performance. Despite the fact that joint ten is actuated, relative to the other schemes 


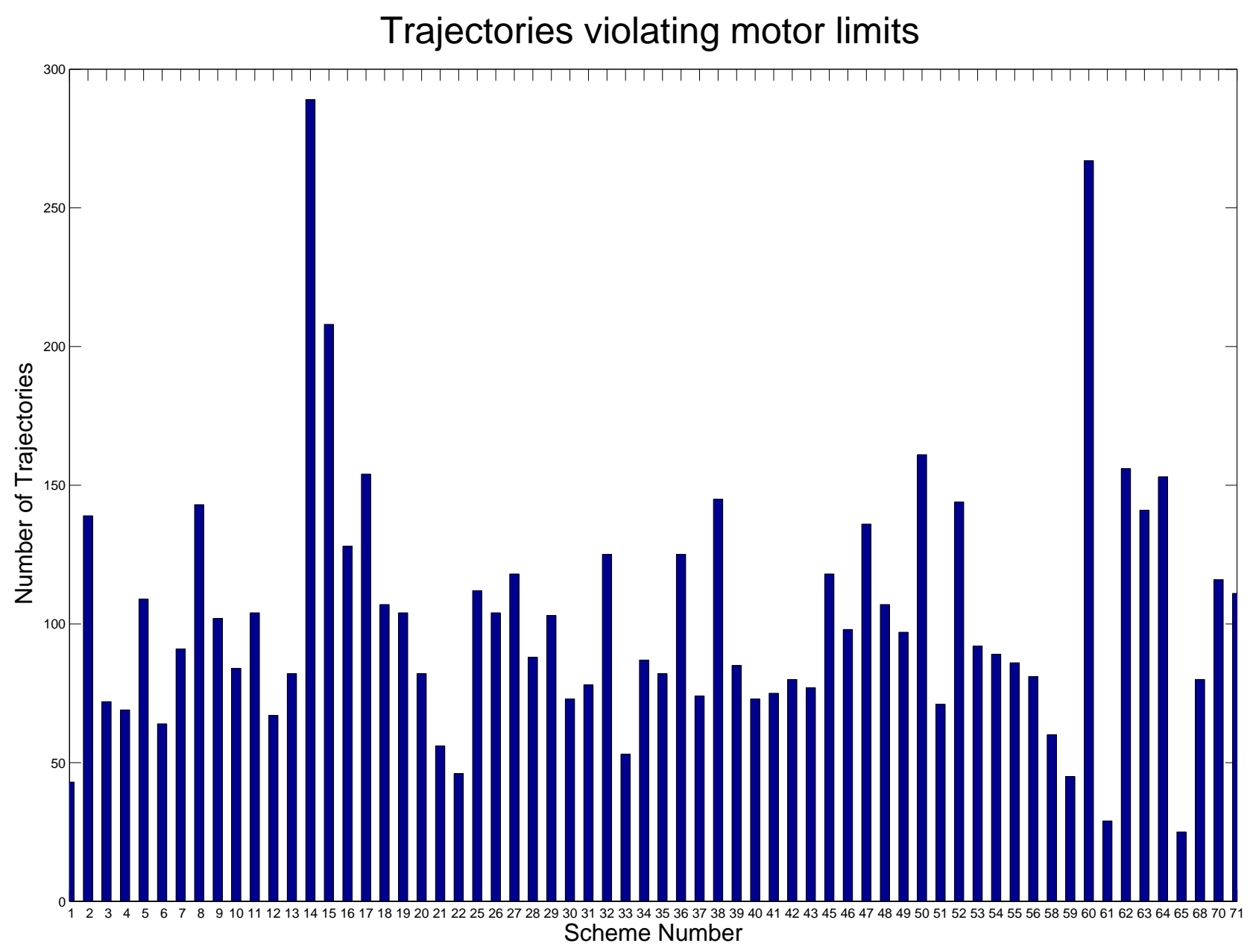

Figure 15: Trajectories that violate torque constraints

the percentage of peak torque violations is low. Furthermore the selected actuated joints, avoid the loss of stiffness associated with the singular configurations. Finally the scheme distributes the motors equally in the two arms.

\section{Conclusion}

This paper presents a study of the NAO robot's two arms engaged in a cooperative task. The DOF of an object, simultaneously grasped by both arms, was explored using screw theory. The infinitesimal motion of the object, for different configurations has been investigated. It has been shown that the nature of this motion may change throughout the workspace due to the relationship between the constraint wrenches. 
The serial singularities of each arm are shown and an investigation into their possible effect on the closed chain system was undertaken. The screw theory analysis demonstrates that the effect of serial singularities in closed loop depends on the actuation scheme that is used. This is validated using a numerical comparison. The constraint singularity due to the closed loop structure is illustrated and the nature of the resulting motion described. The motion is more complex than a loss of stiffness since the arms are simultaneously in a serial singularity configuration. The results from a corresponding numerical analysis are difficult to interpret due to the complexity of this case.

A detailed investigation into the choice of actuated joints was carried out. By considering the wrenches exerted by the actuators on the object, all admissible actuation schemes were enumerated. In addition the schemes considered inadmissible and the causes of this inadmissibility, were illustrated in detail.

The dynamic model of the closed chain system was given. The model was then used to assess the performance with respect to a power loss criterion over many trajectories. This analysis permits the selection of feasible actuation schemes with respect to their dynamic capabilities.

In future work, a control scheme capable of switching the actuation scheme throughout a trajectory will be designed. The objective of this scheme will be to reduce energy loss in the system. Furthermore the behavior of redundant actuation schemes in the closed chain system will be investigated. In addition, the analysis will be extended to articulated and flexible objects.

\section{ACKNOWLEDGEMENTS}

This work was conducted in the framework of the ARMS project. ARMS : A multi arms robotic system for muscle separation, is partly funded by the ANR (Agence Nationale de la Recherche), reference ANR-10-SEGI-000.

\section{References}

[1] Uchiyama, M., and Dauchez, P., 1988. "A symmetric hybrid position/force control scheme for the coordination of two robots". In 1988 IEEE International Conference on Robotics and Automation, Philadelphia, PA, pp. 350-356. 
[2] Chiacchio, P., Chiaverini, S., and Siciliano, B., 1996. "Direct and inverse kinematics for coordinated motion tasks of a two-manipulator system". Journal of Dynamic Systems, Measurement, and Control, 118, p. 691.

[3] Caccavale, F., Chiacchio, P., and Chiaverini, S., 2000. "Task-space regulation of cooperative manipulators". Automatica, 36(6), pp. 879-887.

[4] Yeo, H.-J., Suh, I. H., Yi, B.-J., and Oh, S.-R., 1999. "A single closed-loop kinematic chain approach for a hybrid control of two cooperating arms with a passive joint: an application to sawing task". IEEE Transactions on Robotics and Automation, 15(1), pp. 141-151.

[5] Liu, Y., Xu, Y., and Bergerman, M., 1999. "Cooperation control of multiple manipulators with passive joints". IEEE Transactions on Robotics and Automation, 15(2), Apr., pp. $258-267$.

[6] Cheng, H., Yiu, Y.-k., Member, S., and Li, Z., 2003. "Dynamics and control of redundantly actuated parallel manipulators". IEEE/ASME Transactions on Mechatronics, 8(4), Dec., pp. $483-491$.

[7] Özkan, B., and Özgören, M., 2001. "Invalid joint arrangements and actuator related singular configurations of a system of two cooperating scara manipulators". Mechatronics, 11(4), pp. 491-507.

[8] Long, P., Khalil, W., and Caro, S., 2012. "Control of a lower mobility dual arm system". In International IFAC Symposium on Robot Control, SYROCO, Dubrovnik, Croatia, Vol. 10, pp. 307-312.

[9] Amine, S., Caro, S., Wenger, P., and Kanaan, D., 2012. "Singularity analysis of the h4 robot using grassmann-cayley algebra". Robotica, 30(07), pp. 1109-1118.

[10] Zlatanov, D., Bonev, I., and Gosselin, C., 2002. "Constraint singularities of parallel mechanisms". In Robotics and Automation, 2002. Proceedings. ICRA'02. IEEE International Conference on, Vol. 1, IEEE, pp. 496-502.

[11] Khalil, W., and Kleinfinger, J., 1986. "A new geometric notation for open and closed-loop robots". In Robotics and Automation. Proceedings. 1986 IEEE International Conference on, Vol. 3, IEEE, pp. 1174-1179.

[12] Khalil, W., and Creusot, D., 1997. "Symoro+: a system for the symbolic modelling of robots". Robotica, 15, pp. 153-161. 
P. Long, W. Khalil and S. Caro, submitted to Robotica

[13] Gogu, G., 2007. Structural Synthesis of Parallel Robots: Part 1: Methodology. Springer Verlag.

[14] Long, P., Khalil, W., and Caro, S., 2012. "Kinematic analysis of lower mobility cooperative arms by screw theory". In Proceedings of the 9th International Conference on Informatics in Control, Automation and Robotics, pp. 280-285.

[15] Hunt, K., 1978. Kinematic geometry of mechanisms. Cambridge Univ Press.

[16] Ball, S. R., 1900. A Treatise on the Theory of Screws. Cambridge Univ Pr.

[17] Kong, X., and Gosselin, C., 2007. Type synthesis of parallel mechanisms. Springer Publishing Company, Incorporated.

[18] Amine, S., Tale Masouleh, M., Caro, S., Wenger, P., and Gosselin, C., 2012. "Singularity analysis of 3t2r parallel mechanisms using grassmann-cayley algebra and grassmann line geometry". Mechanism and Machine Theory, 52, pp. 326-340.

[19] O'Brien, J., and Wen, J., 1999. "Redundant actuation for improving kinematic manipulability". In Robotics and Automation, 1999. Proceedings. 1999 IEEE International Conference on, Vol. 2, IEEE, pp. 1520-1525.

[20] Khalil, W., and Dombre, E., 2004. Modeling, identification and control of robots. Butterworth-Heinemann.

[21] Chevallereau, C., Aoustin, Y., et al., 2001. "Optimal reference trajectories for walking and running of a biped robot". Robotica, 19(5), pp. 557-569. 


\section{Appendix}

\section{A Motor Parameters}

Table 4: Motor's specifications

\begin{tabular}{ccccc}
\hline \hline Joint Motor & No load speed(rpm) & Stall Torque $(\mathrm{mNm})$ & Nominal torque $(\mathrm{mNm})$ & Reduction ratio \\
\hline 1 & 11900 & 15.1 & 3.4 & 150.27 \\
2 & 11900 & 15.1 & 3.4 & 173.21 \\
3 & 11900 & 15.1 & 3.4 & 150.27 \\
4 & 11900 & 15.1 & 3.4 & 173.21 \\
5 & 11900 & 15.1 & 3.4 & 50.64 \\
\hline \hline
\end{tabular}

\section{B Dynamic Parameters}

The dynamic parameters of each arm are identical. The inertia tensor of link $j$ is given with respect to frame $j$ as follows:

$$
{ }^{j} \mathbf{I}_{j}=\left[\begin{array}{ccc}
X X_{j} & X Y_{j} & X Z_{j} \\
X Y_{j} & Y Y_{j} & Y Z_{j} \\
X Z_{j} & Y Z_{j} & Z Z_{j}
\end{array}\right]
$$

The first moments of link $j$ are calculated using the mass, denoted as $M_{j}$ and the vector of center-of-mass coordinates denoted as $\mathbf{S}_{j}$, as follows:

$$
M \mathbf{S}_{j}=\left[\begin{array}{lll}
M X & M Y & M Z
\end{array}\right]
$$

The numerical values parameters of the robot are given in Table 5. In the case of the closed chain formulation, the inertial parameters of the extended link 5 , is composed of link 5 , the object and link 10 . 
Table 5: Inertial parameters of the closed chain system

\begin{tabular}{cccccc}
\hline \hline & Link 1(6) & Link 2(7) & Link 3(8) & Link 4(9) & Link 5 \\
\hline $\mathrm{XX}\left(\mathrm{g} \mathrm{mm}^{2}\right)$ & 71025.99 & 82285.13 & 5503.19 & 25194.83 & 122113.5 \\
$\mathrm{XY}\left(\mathrm{g} \mathrm{mm}^{2}\right)$ & -2024.58 & -39780.57 & -22.43 & -2162.93 & 10072.8 \\
$\mathrm{XZ}\left(\mathrm{g} \mathrm{mm}^{2}\right)$ & -17.22 & 7526.01 & -15.34 & 718.46 & 77907.38 \\
$\mathrm{YY}\left(\mathrm{g} \mathrm{mm}^{2}\right)$ & 14057.99 & 290014.459 & 62254.05 & 88903.15 & 558947.02 \\
$\mathrm{YZ}\left(\mathrm{g} \mathrm{mm}^{2}\right)$ & 8.41 & -1529.45 & 5.59 & -108.09 & 5861.52 \\
$\mathrm{ZZ}\left(\mathrm{g} \mathrm{mm}^{2}\right)$ & 73166 & 268423.31 & 63251.24 & 86868.67 & 559976.06 \\
$\mathrm{MX}\left(\mathrm{g} \mathrm{mm}^{2}\right)$ & -1.78 & 18.85 & -25.6 & 25.56 & 63.6 \\
$\mathrm{MY}(\mathrm{g} \mathrm{mm})$ & 24.96 & -5.77 & 0.01 & -2.73 & 1.66 \\
$\mathrm{MZ}(\mathrm{g} \mathrm{mm})$ & 0.18 & 0.65 & -0.19 & 0.96 & 9.54 \\
$\mathrm{M}(\mathrm{g})$ & 69.96 & 123.09 & 59.71 & 77.24 & 333.06 \\
\hline \hline
\end{tabular}

\section{Trajectory Definition}

The trajectory is defined in the joint space, such that from an initial position $\mathbf{q}_{a}^{i}$, a final position $\mathbf{q}_{a}^{f}$ is reached in time $t_{\text {final }}$. The position, velocity and acceleration is calculated as:

$$
\begin{aligned}
& \mathbf{q}_{a}(t)=\mathbf{q}_{a}^{i}+r(t)\left(\mathbf{q}_{a}^{f}-\mathbf{q}_{a}^{i}\right) \\
& \dot{\mathbf{q}}_{a}(t)=\dot{r}(t)\left(\mathbf{q}_{a}^{f}-\mathbf{q}_{a}^{i}\right) \\
& \ddot{\mathbf{q}}_{a}(t)=\ddot{r}(t)\left(\mathbf{q}_{a}^{f}-\mathbf{q}_{a}^{i}\right)
\end{aligned}
$$

The trajectory is calculated from the initial and final conditions:

$$
\mathbf{q}_{a}(t=0)=\mathbf{q}_{a}^{i}, \quad \mathbf{q}_{a}\left(t_{\text {final }}\right)=\mathbf{q}_{a}^{f}, \quad \dot{\mathbf{q}}_{a}(t=0)=\dot{\mathbf{q}}_{a}\left(t_{\text {final }}\right)=0, \quad \ddot{\mathbf{q}}_{a}(t=0)=\ddot{\mathbf{q}}_{a}\left(t_{\text {final }}\right)=0
$$

$r(t)$ is the $5^{t h}$ degree polynomial interpolation function calculated as:

$$
r(t)=10\left(\frac{t}{t_{\text {final }}}\right)^{3}-15\left(\frac{t}{t_{\text {final }}}\right)^{4}+6\left(\frac{t}{t_{\text {final }}}\right)^{5}
$$

